\title{
Crustal geomagnetic field and secular variation by regional and global models for Austria
}

\author{
Klaudio PEQINI'*), Bejo DUKA ${ }^{11}$, Ramon EGL(2) \& Barbara LEICHTER ${ }^{2)}$ \\ 1) University of Tirana, Faculty of Natural Science, blvd. Zogu I, Nr. 25, Tirana, Albania; \\ 2) Zentralanstalt für Meteorologie und Geodynamik, ZAMG, 1190 Vienna, Hohe Warte 38; \\ *) Corresponding author: klaudio.peqini@fshn.edu.al
}

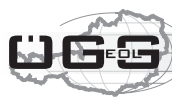

KEYWORDS crustal field; regional model; global model; polynomial approximation; magnetic field anomalies

\begin{abstract}
Using 12-year-long series of data (2001-2012) from geomagnetic observatories and repeat stations in Austria and its neighboring countries, a regional spatial-temporal (ST) model is developed based on the polynomial expansion consisting of latitude, longitude, and time of the geomagnetic field components and total magnetic field $F$. Additionally, we have used three different global models (CHAOS-5, POMME-9, and EMM2015), which are built on spherical harmonics up to a maximum degree $L_{\max }$ and give the core field and crustal field separately. The normal field provided by the ST model and its "model bias", which comprise the residuals of the differences between measured and predicted values, are calculated and the respective maps are shown. The residuals are considered an estimate of the local crustal field. In the case of global models, we have applied for each of these three methods to calculate the "model bias": residuals of the differences between observed values and predicted values of the model, residuals of the differences between observed values and core field values of the model, and the average bias for the period 2001-2012. The normal field of the region of Austria provided by each global model is also calculated. Generally, the regional and global models yield relatively similar crustal fields for the Austrian region, especially when the first method is used. The normal fields calculated by them are in good agreement with each other. Each of the global models directly provides the crustal field, and they are compared with the aeromagnetic data provided by aeromagnetic surveys over the Austrian region. The ST model is in better agreement with aeromagnetic data. We have also analyzed the secular variation over the region, which is calculated from the rate of change of normal field given by the ST and global models.
\end{abstract}

\section{Introduction}

The geomagnetic field originates from two different sources: the internal sources located inside the Earth, and the external sources located in the magnetosphere and ionosphere. The external sources are directly connected to the solar wind (though there are other factors that influence the dynamics), and there are elaborate models that describe the dynamics of the magnetic field generated by them (Jacobs, 1991; Voigt, 1981, Olsen and Stolle 2016). The main internal source is the convective motion of the electrically conductive fluid in the outer core, which is essential for the functioning of the geodynamo. This mechanism creates the main magnetic field, whose intensity (total field $F$ ) varies slowly with time, typically at a rate of $70-80 \mathrm{nT} / \mathrm{yr}$. The second most important internal source is related to the geological structure of the crust, and it creates the lithosphere field. The main part of the lithosphere (crustal) field is produced by the remanent magnetization of rocks located in the crust and uppermost mantle, acquired during cooling below the Curie temperature of magnetic minerals (Lowrie, 2007). There is a minor part of the lithospheric magnetization that is induced, being locally (roughly) parallel to, and proportional to, the ambient field (Lowrie, 2007). By virtue of the fact that the main field is several orders of magnitude larger than the field of other contributions, it is quite expectable that this induced crustal field varies with the time scale of the main field (Maus and Haak, 2002). The remanent magnetization component of the crustal field can be considered constant in the decadal time scale considered in this study. It is a common assumption in global magnetic field modeling that the crustal field is static (Macmillan and Thomson, 2003).

The main goal of this paper is the investigation of crustal field contributions over the small and elongated region of Austria without distinction between induced and remanent components. For this purpose, repeat station and geomagnetic observatory data from Austria, as well as from neighboring countries, over the period 2000-2012 are considered. In order to recover crustal contributions from the observed data, two types of models are used, namely, a regional model and three different global models.

Regional models are based on denser data grids than global models over a limited region and therefore produce a more detailed picture of the magnetic field in those regions than the global models (Haines, 1985). Considering the potential nature of the field, some methods of regional modeling use mathematical expansions of the geomagnetic potential in harmonics, such as rectangular harmonic analysis (RHA) (Alldredge, 1981; Nakagawa and Yukutake, 1985), and spherical cap harmonic analysis (SCHA) (De Santis et al., 1990). Other 
methods use the representation of the regional geomagnetic field by their equivalent sources (equivalent source inversion [ESI]), such as equivalent dipole sources (Mayhew, 1979, Purucker, 1990), monopoles (O'Brien and Parker, 2004), and spherical prisms (Asgharzadeh et al., 2008), or by surface polynomials. Comparison of RHA and its modified version, namely, rectangular polynomial analysis (RPA), with SCHA for 42 observatories of Europe provided best results for SCHA (Düzgit and Malin, 2000). The comparison of different methods (SCHA, RHA, and ESI dipoles or monopoles) applied to fit the satellite data from the Italian region showed that the best result is achieved by SCHA (Duka et al., 2004). However, SCHA does not provide reliable results when the spherical cap aperture is $<6^{\circ}$. Therefore, we cannot apply this method for the Austrian region (spherical cap about $2^{\circ}-3^{\circ}$ ).

Very long-wavelength magnetic anomalies (>600 km) have been revealed by near-Earth satellite missions, e.g., MagSat. These magnetic anomalies are modeled by the ESI method (Taylor and Ravat, 1995; Nolte and Hahn, 1992). Dipole schemes can be used to fit the MagSat magnetic anomalies over the Austrian region. However, this method proved to be unstable over the Austrian region, although the method was successful when applied on Southeast Asia (Achache et al., 1987). It seems that the failure of the method in this case is due to the small extension of the region. Therefore, the only method available for a small-scale regional model is based on fitting the magnetic components with polynomials.

Another aim of this paper is to study the secular variation (SV) over the considered region. The global models (POMME-9, EMM2015, and CHAOS-5) and the regional ST model provide the SV by calculating the differences between the consecutive annual means for each component of the magnetic field and the total field $F$. At first, the regional and global models are discussed, and the various methods used to calculate the crustal field with the regional and global models are explained. Then, a detailed account of the data used is given and the regional model is described with some of the results obtained by this model, followed by some important details on the global models and the exposition of the results obtained by the global models. In the following section, the results of the SV calculations are presented, followed by discussions and conclusions.

\section{Regional models and global models}

Fitting the geomagnetic data by surface polynomials is straightforward, but these polynomials do not include a radial term, thus the data obtained at different altitudes cannot be considered simultaneously (Haines, 1995). The surface polynomial method involves the expansion of the geomagnetic field components in analytical functions such as polynomials. There are two kinds of surface polynomial models: (1) constrained polynomials, for which, in the source-free region, the magnetic field is considered to have zero curl and, consequently, only the $Z$ component; and (2) unconstrained polynomials.
Because the polynomials lack any altitude dependence, only the $z$ component of the curl of the geomagnetic field can be used as a constraint. The fact that we could not use the other components of the curl as constraints led us to choose unconstrained polynomials to construct the ST model (see Section 3).

The regional model, named hereafter as the normal field model, approximates the geomagnetic field components by polynomials of certain order in latitude and longitude. This approach is commonly used in many countries to recover the local anomalies of the geomagnetic field with crustal origin. We interpret the model residuals (see following text) as the local crustal contribution to the geomagnetic field, although there might be minor contributions from the geographical relief, which - in our case - is very difficult to quantify.

In order to obtain the crustal field separately from the regional model, we have used three different global models based on spherical harmonics (CHAOS-5, POMME-9, and EMM2015) to calculate the geomagnetic field at the points where measurements are available (observatories or repeat stations). We calculate the crustal field using three methods. The first method involves calculating the residuals between the measured values of each of the components and the total field with the corresponding values generated by each of the global models. The resulting residuals should represent, in principle, mainly the crustal field.

The global models can give the magnetic field up to a given degree of spherical harmonics. For the implementation of the second method, we calculate the magnetic field truncated to its core part, i.e., up to degree and order 13 , yielding the geomagnetic field originating from the core (see Section 5). Then, we calculate the residuals between the measured values for each component and the total field with the corresponding values of the core field generated by the global models.

The third method takes into consideration the fact that the crustal field is mainly static. Therefore, we initially calculate the residuals as in the first method for every year of the period 2001-2012. Thus, for every component, as well as the total field, and for each repeat station in Austria, we have 12 residuals. Then, we calculate the averages for these residuals and consider them to be the signature of the crustal field.

The so-called "crustal bias" (Mandea and Langlais, 2002) is estimated by comparing the magnetic components measured in the observatory with the core field predicted by a given global models. These models are based only on satellite data sets (Bloxham and Gubbins, 1986), wherein the contributions of the crustal sources of small wavelength are negligible. Therefore, the residuals between observed values and model values represent the overwhelming majority of the crustal field of induced and remanent origin. When a global model makes use of observatory data, it can reproduce almost the entire crustal field. Here, we study a kind of "bias", which is estimated as the difference in the values of the geomagnetic field components 
observed at a given observatory or repeat station at a given epoch with the respective values predicted by the geomagnetic field models based on ground and satellite data.

Different models provide different "biases" (named "model bias") at the same observatory/repeat station. Subtraction of time-averaged "biases" (e.g., for the period 2001-2012) from the corresponding time series does not completely eliminate the contribution of external sources (Duka et al., 2016). However, it turns out that the external contribution is constant (some nanoteslas) for all models and almost the same in every place. Therefore it is possible to apply the last method to estimate the geomagnetic crustal field. We subtracted the corresponding values of the core field predicted by a geomagnetic model from the magnetic components measured in an observatory/repeat station (up to degree 14 for the POMME-9 model, up to degree 16 for the EMM2015 and up to degree 20 for the CHAOS-5 model).

\section{The data}

Austria has a magnetic observatory (Wien Kobenzl until 2016 and Conrad Observatory since 2012) and a well-maintained repeat station network with 14 points (Fig. 1). Repeat station measurements are reduced to the middle of each year. These data are integrated with analogous measurements by neighboring countries. Observatory data for Budkov (BDV), Fürstenfeldbruck (FUR), Hurbanovo (HRB), Nagycenk (NCK), and Tihany (THY) have been obtained from INTERMAGNET (www. intermagnet.org). Repeat station data from Germany, Czech Republic, and Hungary are available from the World Data Center (WDC), hosted by the British Geological Survey (http://www.geomag.bgs.ac.uk/data_ service/data/surveydata.shtml).

The number of repeat stations is different for different epochs. There are years such as 1997 and 2006 when the numbers of measurement points and repeat stations or observatories that provide data are, respectively, 50 and 43. In other time intervals, there are less data. However, the network of repeat stations in Austria (14 points) provides data for the whole period under study, i.e., 20012012 , enabling us to use the third method for calculating the residuals. We have also included data from the year 1997, which enhance the stability of the ST model. However, we have not calculated the residuals for this epoch because they lie outside the validity period for all the global models. Thus, the number of data for each component and the total field is 387.

All data used for the models are reduced to the specific time by standard methods. To cope with the lack of height in the model's equations, all data have been reduced to two different altitudes: 0 and $3 \mathrm{~km}$, by using the dipolar approximation (www-gpsg.mit.edu/12.201_12.501/
BOOK/chapter3.pdf) near the Earth's surface. The reduction to $3 \mathrm{~km}$ altitude (Fig. 1) enables us to compare our results with the results of aeromagnetic measurements performed at the same altitude.

\section{The ST model of SVs}

\subsection{The model}

An ST model for Austria is constructed by polynomial regression of the measurements reduced to midyear epochs between 2001 and 2012, the colatitudes comprised between $40^{\circ} \mathrm{N}$ and $45^{\circ} \mathrm{N}$, and the longitudes were between $8^{\circ} \mathrm{E}$ and $18^{\circ} \mathrm{E}$. The magnetic field is expressed by its components $X$ along the geographic North, $Y$ along the East, and $Z$ downward.

Let $\boldsymbol{y}$ be the vector containing all data for one magnetic component $(X, Y$, or $Z)$ reduced to a given time. The normal field at the location of the $i$-th measurement is given by the following polynomial expression:

$$
\begin{aligned}
y_{i}=a & +b\left(\varphi_{i}-\varphi_{c}\right)+c\left(\lambda_{i}-\lambda_{c}\right)+d\left(\varphi_{i}-\varphi_{c}\right)\left(\lambda_{i}-\lambda_{c}\right) \\
& +e\left(\lambda_{i}-\lambda_{c}\right)^{2},
\end{aligned}
$$

where $\varphi_{i}$ and $\lambda_{i}$ are the colatitude and longitude of the $i$-th measurement, $\varphi_{c}=42.5^{\circ} \mathrm{N}$ and $\lambda_{c}=13^{\circ} \mathrm{E}$ are the colatitude and longitude of a central point of the selected area, and $a, b, c, d$, and $e$ are the model coefficients for the given time to be determined by least-squares regression. Because of the principal E-W extension of the investigated area, spanning over $>10^{\circ}$ and only extending north-south (N-S) over $\sim 5^{\circ}$, the polynomial in Eq. (1) is of the first order with respect to latitude and second order with respect to longitude. The time dependence of the field is accounted for by a polynomial expansion of each of the coefficients in Eq. (1) with respect to time $t$, for instance, refer the following expression

$$
a(t)=a_{0}+a_{1} t+a_{2} t^{2}
$$

for coefficient $a$, where $t$ is specified as the modified Julian date (mjd2000) with Day 1 being the 1 st of January 
2000. Inserting Eq. (2) and the analogous expressions for the other coefficients into Eq. (1) and rearranging terms, we obtain the following:

$$
\begin{aligned}
y_{i}=a_{0} & +b_{0}\left(\varphi_{i}-\varphi_{c}\right)+c_{0}\left(\lambda_{i}-\lambda_{c}\right) \\
& +d_{0}\left(\varphi_{i}-\varphi_{c}\right)\left(\lambda_{i}-\lambda_{c}\right)+e_{0}\left(\lambda_{i}-\lambda_{c}\right)^{2} \\
& +a_{1} t+b_{1} t\left(\varphi_{i}-\varphi_{c}\right)+c_{1} t\left(\lambda_{i}-\lambda_{c}\right) \\
& +d_{1} t\left(\varphi_{i}-\varphi_{c}\right)\left(\lambda_{i}-\lambda_{c}\right)+e_{1} t\left(\lambda_{i}-\lambda_{c}\right)^{2} \\
& +a_{2} t^{2}+b_{2} t^{2}\left(\varphi_{i}-\varphi_{c}\right)+c_{2} t^{2}\left(\lambda_{i}-\lambda_{c}\right) \\
& +d_{2} t^{2}\left(\varphi_{i}-\varphi_{c}\right)\left(\lambda_{i}-\lambda_{c}\right)+e_{2} t^{2}\left(\lambda_{i}-\lambda_{c}\right)^{2} .
\end{aligned}
$$

This is a system of $N=387$ equations for each component as well as for the total field $F ; y_{i}$ is initially the vector of data for the $X$ component, then for the $Y$ and $Z$ components and for the total field $F$. There are 15 unknowns (the coefficients) for each component and $F$, and each system of equations can be expressed in matrix form as follows:

$$
\boldsymbol{y}=A \boldsymbol{x}
$$

where the matrices involved in this equation are from left to right: the vector of data $(N \times 1)$, the ST matrix $(N \times 15)$, and the vector of coefficients $(15 \times 1)$. Consequently, the vector $\boldsymbol{x}$ is calculated using the following expression:

$$
\boldsymbol{x}=A^{-1} \boldsymbol{y},
$$

The differences $\boldsymbol{y}^{\text {measured }}-\boldsymbol{A} \boldsymbol{x}$ between the measured values of the components and the corresponding values obtained by the ST model are considered to be the signature of the crustal field at the measurement locations. Such differences are mainly due to the remanent and induced magnetization of the local geological structures in the upper crust. The effect of topography can be considered negligible (Chiappini et al., 2000; Khesin et al., 1996). There can possibly be a contribution from components of the external field, which is not modeled by the global models (Duka et al., 2016). It should not exceed a few nanoteslas, and it is expected to be homogeneous over small regions such as the one considered here. Least-squares solutions of Eq. (5) have been obtained with built-in routines of MATLAB 2011a after Richter (1995). The standard errors of the estimated ST model coefficients are given by the diagonal elements of the correlation matrix $C=\left(A^{T} A\right)^{-1}$.

\subsection{Results of the ST model of Austria}

The ST model results for $0 \mathrm{~km}$ altitude are listed in Table 1. The model coefficients for the $3 \mathrm{~km}$ altitude are almost identical. All coefficients corresponding to quadratic terms in time are extremely small, meaning that SV is almost linear in time for the considered period. Coefficient errors are also very small, indicating that the model is stable.
Klaudio PEQINI, Bejo DUKA, Ramon EGLI \& Barbara LEICHTER

\begin{tabular}{lcccc} 
& $\mathbf{X}$ & $\mathbf{Y}$ & $\mathbf{Z}$ & $\boldsymbol{\sigma}_{\text {coeff }}$ \\
\hline$a_{0}$ & 21133.03 & 513.43 & 42642.33 & 0.121 \\
$b_{0}$ & 543.34 & 10.68 & -587.91 & 0.161 \\
$c_{0}$ & -16.56 & 93.37 & 74.21 & 0.052 \\
$d_{0}$ & -5.53 & -1.30 & 5.38 & 0.072 \\
$e_{0}$ & 2.13 & -0.70 & 0.80 & 0.017 \\
$a_{1}$ & $1.64 \times 10^{-02}$ & $9.41 \times 10^{-02}$ & $9.06 \times 10^{-02}$ & $9.33 \times 10^{-05}$ \\
$b_{1}$ & $-1.48 \times 10^{-02}$ & $2.13 \times 10^{-03}$ & $9.76 \times 10^{-04}$ & $1.29 \times 10^{-04}$ \\
$c_{1}$ & $-8.59 \times 10^{-04}$ & $-1.61 \times 10^{-03}$ & $-7.64 \times 10^{-05}$ & $4.25 \times 10^{-05}$ \\
$d_{1}$ & $3.28 \times 10^{-03}$ & $-1.05 \times 10^{-03}$ & $3.80 \times 10^{-04}$ & $6.13 \times 10^{-05}$ \\
$e_{1}$ & $-4.39 \times 10^{-04}$ & $-4.20 \times 10^{-04}$ & $2.34 \times 10^{-04}$ & $1.45 \times 10^{-05}$ \\
$a_{2}$ & $1.09 \times 10^{-06}$ & $3.85 \times 10^{-06}$ & $-1.53 \times 10^{-06}$ & $2.58 \times 10^{-08}$ \\
$b_{2}$ & $2.86 \times 10^{-06}$ & $-1.68 \times 10^{-07}$ & $-2.79 \times 10^{-07}$ & $2.74 \times 10^{-08}$ \\
$c_{2}$ & $-6.15 \times 10^{-08}$ & $1.16 \times 10^{-07}$ & $2.53 \times 10^{-07}$ & $9.81 \times 10^{-09}$ \\
$d_{2}$ & $-3.99 \times 10^{-07}$ & $2.51 \times 10^{-07}$ & $-6.37 \times 10^{-08}$ & $1.25 \times 10^{-08}$ \\
$e_{2}$ & $8.72 \times 10^{-08}$ & $4.52 \times 10^{-08}$ & $-1.00 \times 10^{-07}$ & $3.06 \times 10^{-09}$ \\
\hline
\end{tabular}

Table 1: Values of the coefficients of the ST model for the data set with reduction at $3 \mathrm{~km}$.

Maps for each field component and the total field $F=\sqrt{X^{2}+Y^{2}+Z^{2}}$ have been generated with Eq. (3) and the coefficients of Table 1 on a regular $0.25^{\circ} \times 0.25^{\circ}$ grid. Here, we present the results for two epochs, year 2003 and 2006. We discuss two models: one derived by the data set reduced at $0 \mathrm{~km}$ altitude and the other derived by the data set reduced at $3 \mathrm{~km}$ altitude. In Figs. 2 and 3, the isolines of the normal field (ST model) are plotted by dotted lines and the isolines of the residuals (local anomalies, i.e., crustal field) are plotted by solid lines. According to the maps (Figs. 2 and 3), there are small changes between the residuals for different years; however, some patterns are visible in both cases located in the center and the east of the area under study. These features should be contributed by the remanent magnetization of the local anomalies (crustal field). The anomalies recovered for the data set reduced at $0 \mathrm{~km}$ are almost identical to their counterparts shown in Fig. 3, emphasizing the crustal nature of these features. Hence, from here on, we will discuss only the results for the data set reduced at $3 \mathrm{~km}$ altitude.

The impact of data quantity on the quality of the crustal field recovery is visible (Figs. 2 and 3). The year 2006 has the most data, and the local anomalies in the eastern and the central part of Austria are captured. The comparison with the anomalies recovered from aeromagnetic measurements (Fig. 1) shows good agreement. The year 2003 has slightly fewer data than 2006, and this explains why the anomaly in Southeastern Austria for the former epoch is less clear. In the years with less data, recovery of the anomalies is distorted. The effect of the nonhomogeneous distribution of the data could not be removed even by altering the time dependence in Eq. (3). In the linear dependence case, the results are very similar compared to the actual model.

\section{Crustal field derived from the global models}

\subsection{The models}

The global models considered in this study describe the components of the geomagnetic field, where each of them is expanded into spherical harmonics series. 


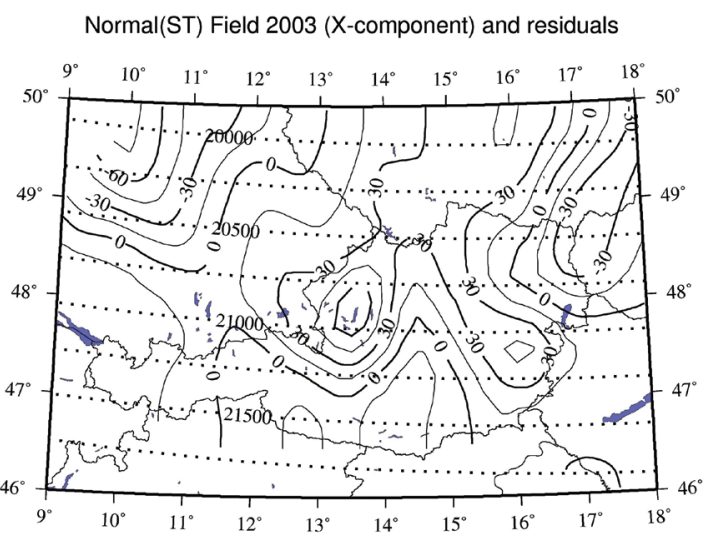

Normal(ST) Field 2003 (Y-component) and residuals

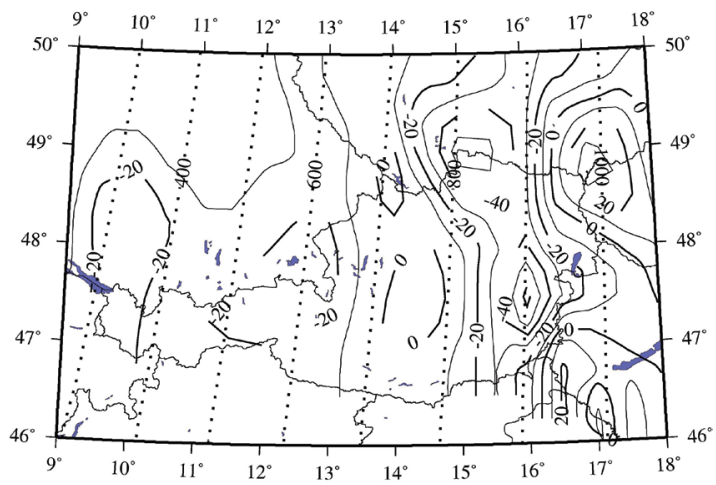

Total Normal(ST) Field 2003 (F) and residuals

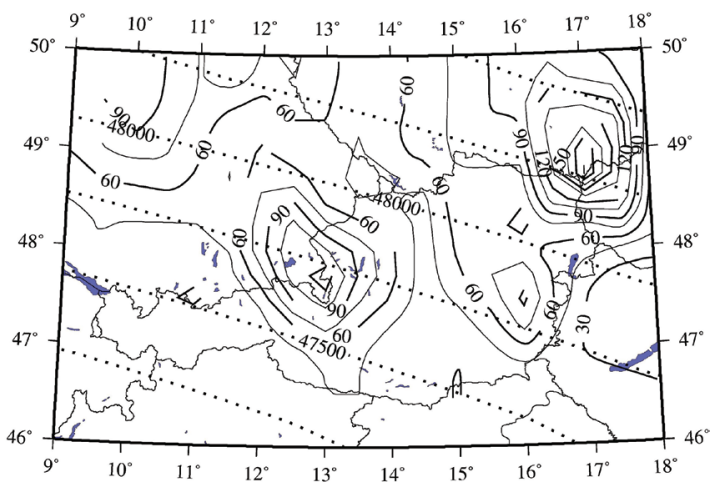

Figure 2: $X, Y, Z$, and $F$ maps of Austria for 2003 for the data set reduced at $3 \mathrm{~km}$, including the normal field (dotted lines) and residuals (solid) isolines. Data are in nanoteslas (nT).
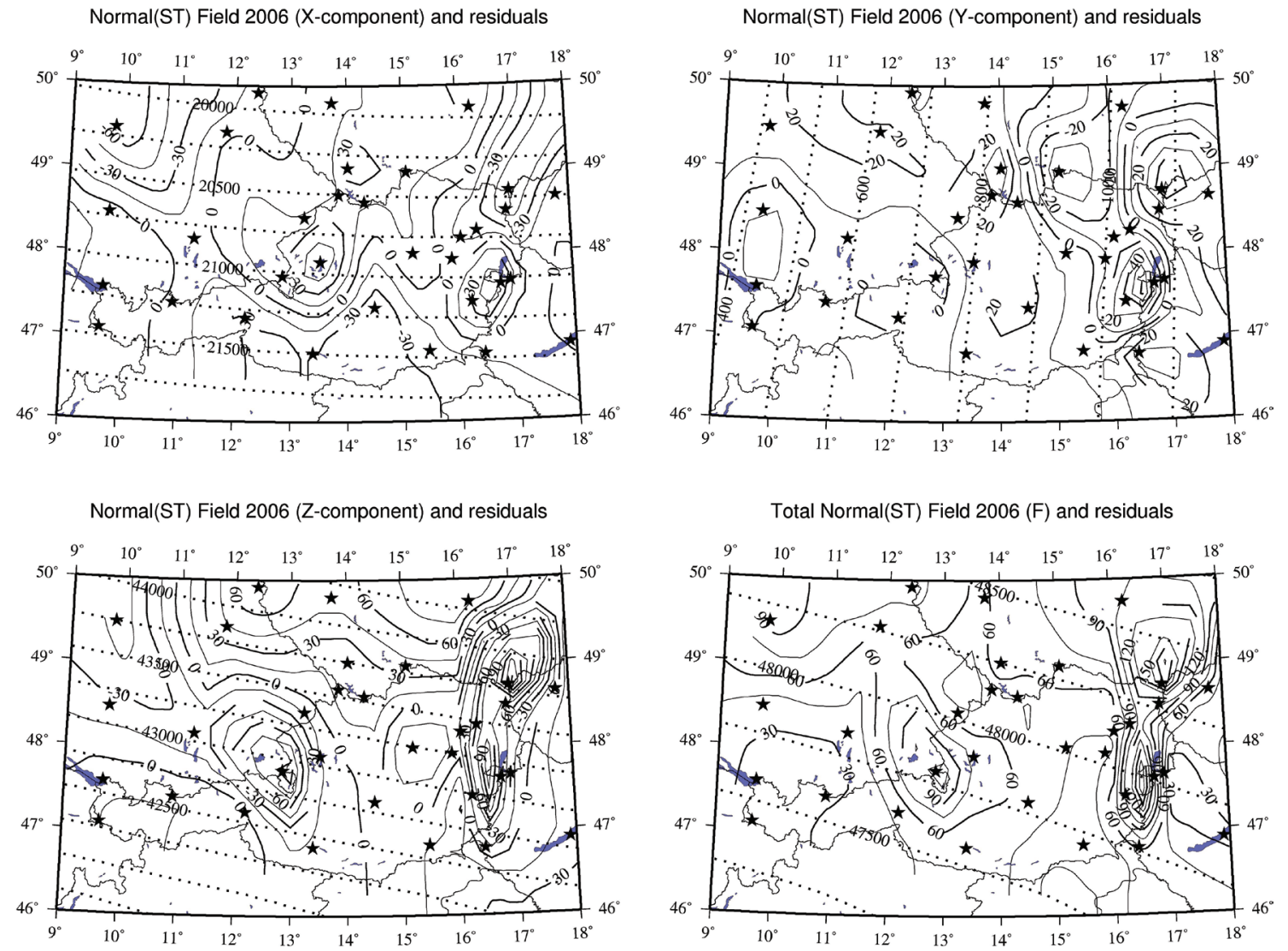

Figure 3: $X, Y, Z$, and $F$ maps of Austria for 2006 for the data set reduced at $3 \mathrm{~km}$, including the normal field (dotted lines) and residuals (solid) isolines. Stars mark the observatories/repeat stations. Data are in nanoteslas (nT). 
The scalar coefficients of these series are known as the Gauss coefficients. These quantities are determined from a given set of data that span a certain period of time, i.e., the validity period of the respective global model (Backus et al., 1996). In theory, an infinity of Gauss coefficients is needed to fully describe the geomagnetic field. In practice, the expansion into the spherical harmonics series is truncated up to a maximum degree $L_{\max }$. The higher the value of the maximum degree, the more accurate is the global model (Duka et al., 2016).

Usually, the models provide separately the main field (core field) up to a degree $L_{c}=13-14$, as well as the static lithospheric field (uppermost crust) beyond $L_{c}$. However, the value of $L_{c}$ varies from model to model depending on the structure of each of them and may exceed $L_{c}=13$. The global models used in this paper have the following characteristic degrees, $L_{\max }=133$ and $L_{c}=13$ (POMME-9); $L_{\text {max }}$ $=720$ and $L_{c}=15$ (EMM2015, Maus, 2010); and $L_{\max }=95$ and $L_{c}=20$ (CHAOS-5, Finlay et al., 2015).

The residuals calculated for each component and the total field are shown in Figs. 4-7. The residuals are calculated as measured values minus the values given by the global model. The maps for the respective components are arranged in individual figures, making the comparison easier.

The maps for the $X$ component show two anomalies in the central and southeastern region of Austria, although they are not very pronounced. On the other hand, the maps for the residuals that pertain to the $Y$ component are quite similar (Fig. 5). There are three distinct circular shapes, i.e., anomalies, in the right part of each of the maps. Very similar are the maps for the $Z$ component (Fig. 6). In this case, there appear three anomalies, of which two are located in eastern Austria, while the third anomaly is located close to the center of each of the maps. The anomalies seem to be more pronounced in the case of the EMM2015 model. The similarities in all cases are both qualitative and quantitative. The $Z$ component is comparable to the total field and, consequently, the maps for $F$ should be very similar to those for $Z$ (Fig. 7). The discrepancies between models are expectable due to the inner structure of the models that are found in Duka et al. (2016).

The quality of maps is heavily reduced in those years for which only few observational data are available. The maps for these years do not allow the drawing of any valuable interpretation or conclusion because the field lines are disrupted and none of the magnetic field anomalies are distinguishable. Therefore, we skip them in our analysis and focus mostly on the year 2006 because, for this year, there are more data, which allows the reconstruction of the crustal field with higher accuracy. The maps for the other years (such as 2003) with numerous data show small discrepancies due to the smaller amount of data at our disposal. Very similar results are obtained for the data reduced at the altitude of $0 \mathrm{~km}$ (not shown here).
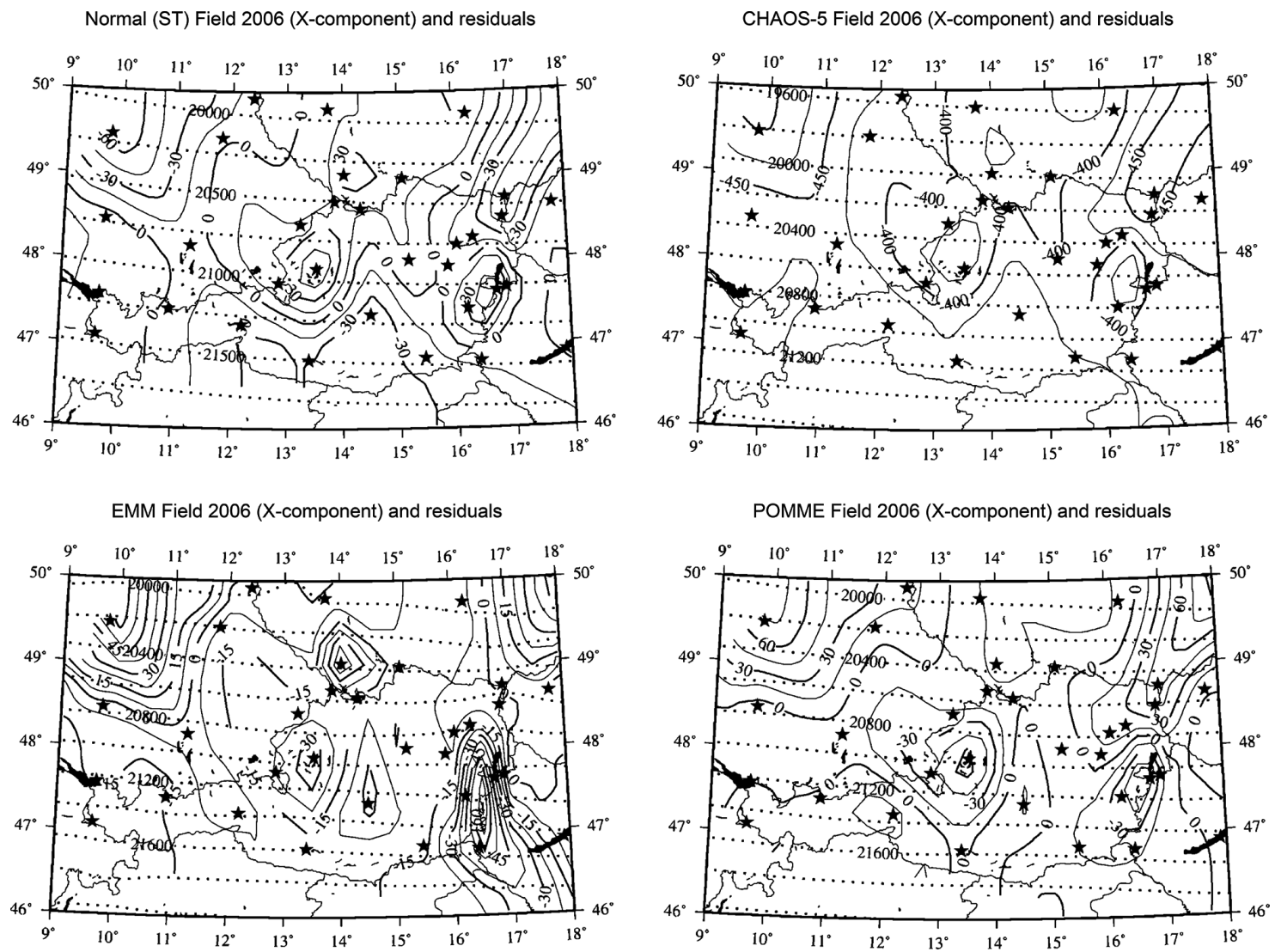

Figure 4: The residuals for the $X$ component at the $3 \mathrm{~km}$ altitude recovered by (clockwise) the ST model, CHAOS-5 model, POMME- 9 model, and EMM2015 model, including the normal field (dotted lines) and residuals (solid) isolines. The data are in nanoteslas (nT). Observatories and repeat stations are marked with black stars. 
Normal (ST) Field 2006 (Y-component) and residuals

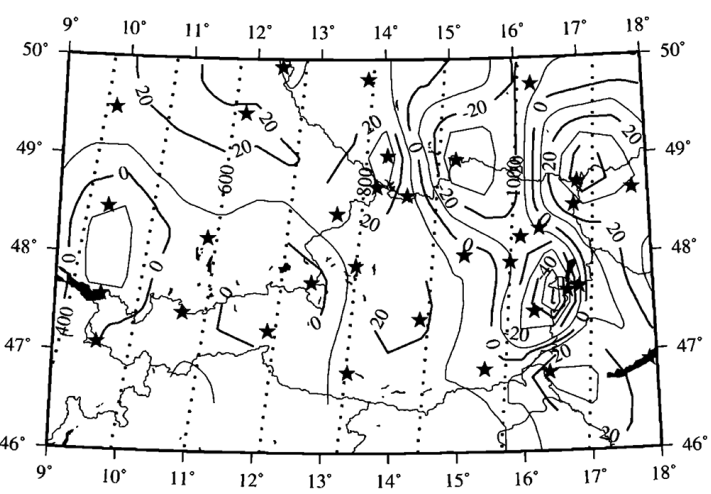

EMM Field 2006 (Y-component) and residuals

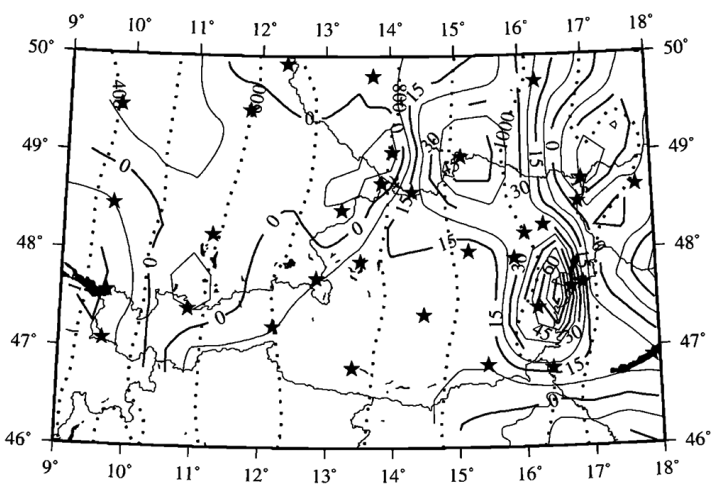

CHAOS-5 Field 2006 (Y-component) and residuals

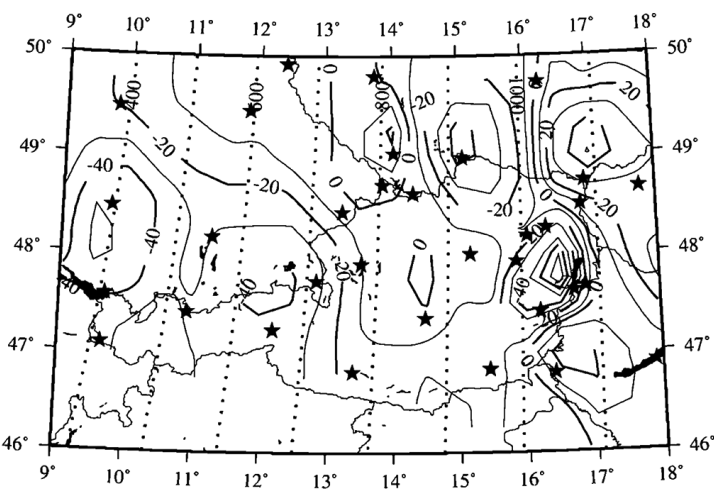

POMME Field 2006 (Y-component) and residuals

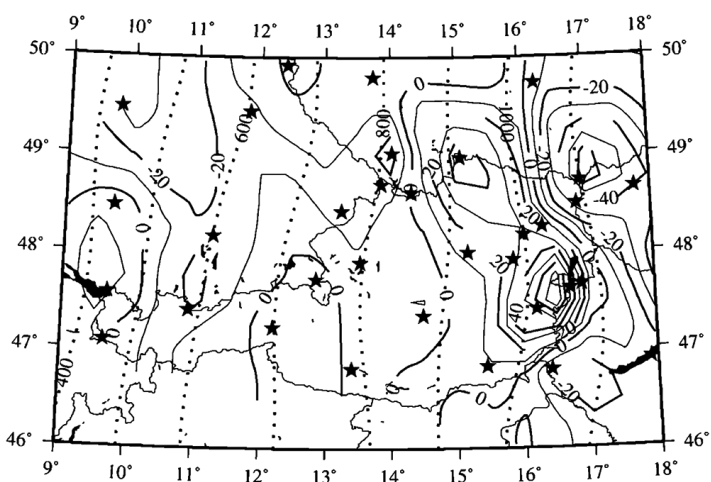

Figure 5: The residuals for the $Y$ component at the $3 \mathrm{~km}$ altitude recovered by (clockwise) the ST model, CHAOS-5 model, POMME-9 model, and EMM2015 model, including the normal field (dotted lines) and residuals (solid) isolines. The data are in nanoteslas (nT). Observatories and repeat stations are marked with black stars.

Normal (ST) Field 2006 (Z-component) and residuals

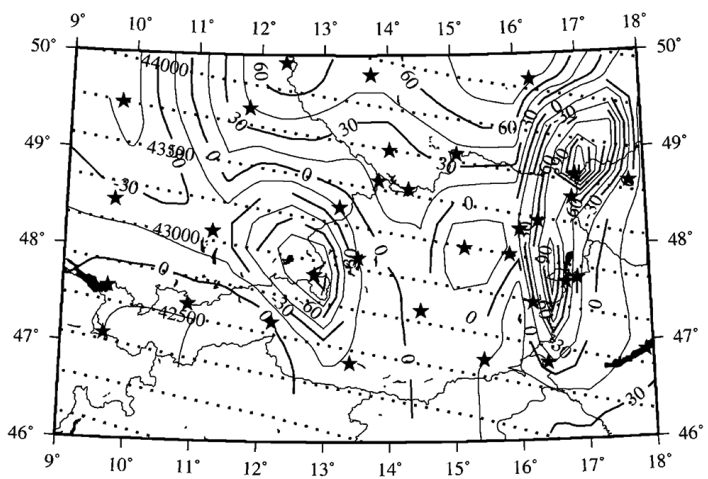

EMM Field 2006 (Z-component) and residuals

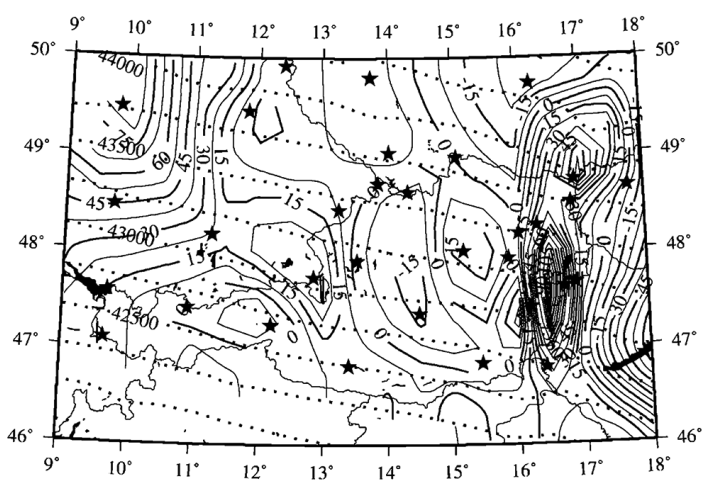

CHAOS-5 Field 2006 (Z-component) and residuals

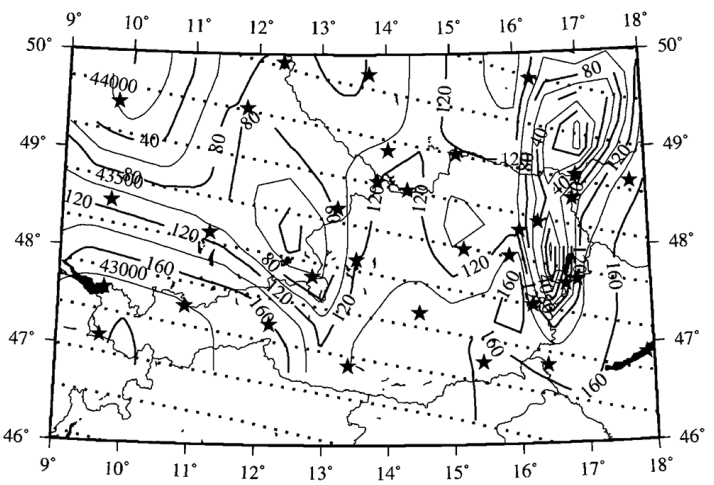

POMME Field 2006 (Z-component) and residuals

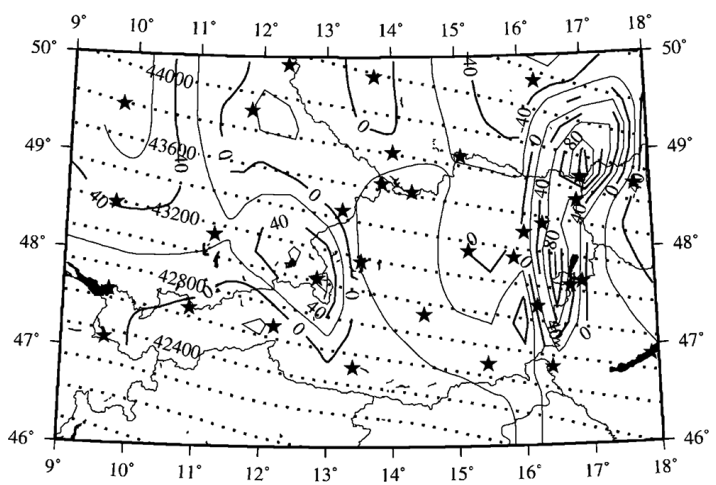

Figure 6: The residuals for the $Z$ component at the $3 \mathrm{~km}$ altitude recovered by (clockwise) the ST model, CHAOS-5 model, POMME-9 model, and EMM2015 model, including the normal field (dotted lines) and residuals (solid) isolines. The data are in nanoteslas (nT). Observatories and repeat stations are marked with black stars. 

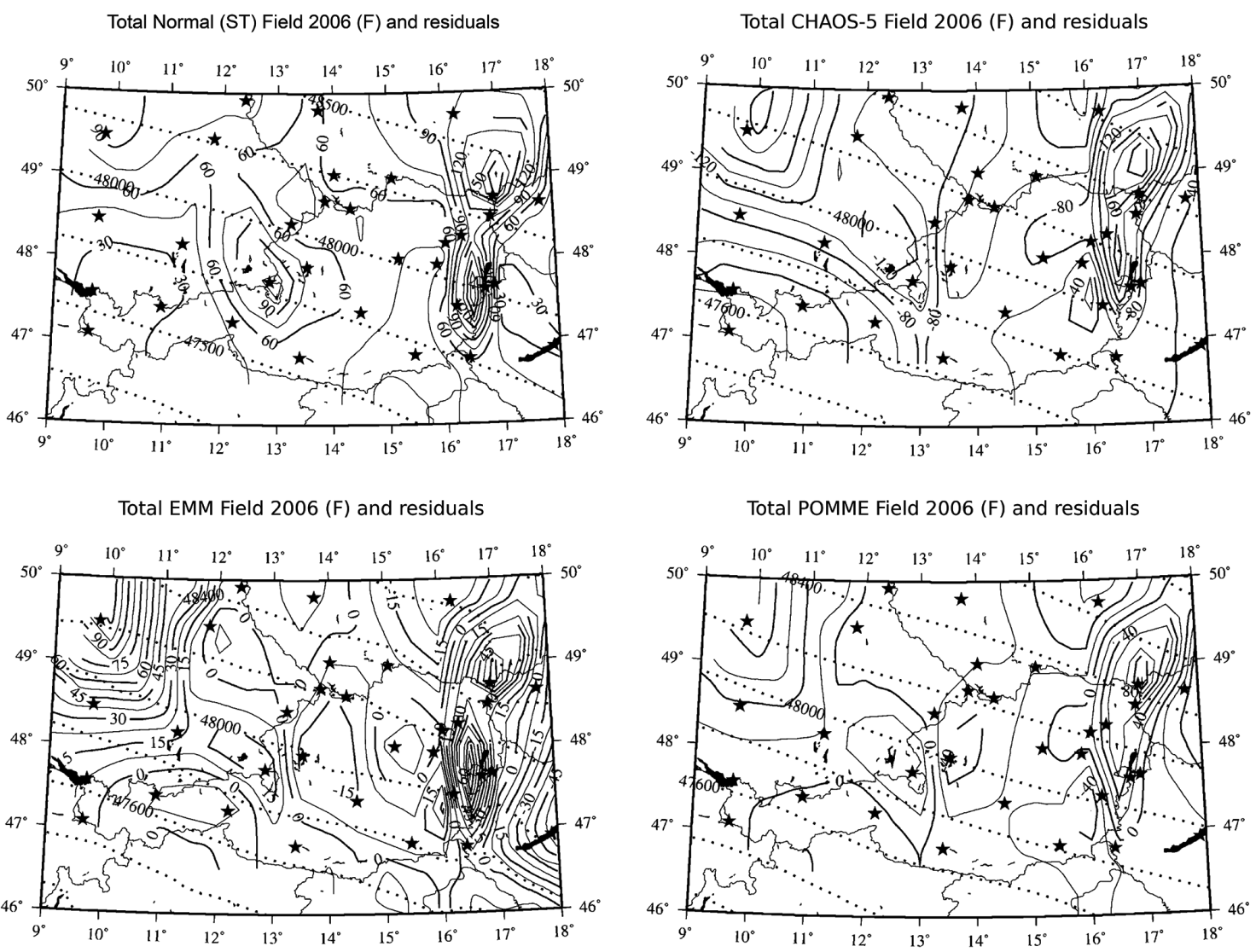

Figure 7: The residuals for the total field $F$ at the $3 \mathrm{~km}$ altitude recovered by (clockwise) the ST model, CHAOS-5 model, POMME-9 model, and EMM2015 model, including the normal field (dotted lines) and residuals (solid) isolines. The data are in nanoteslas (nT). Observatories and repeat stations are marked with black stars.

We noticed that the anomalies for almost all components and all models are intensified especially in the eastern part of the country, while the patterns of the model field (dotted lines) are almost the same. The total field $F$ for all the models actually shows very similar features to that found for the $Z$ component, and the magnitudes of the magnetic field in both eastern anomalies range from $90 \mathrm{nT}$ to $120 \mathrm{nT}$. The central anomaly is also visible and the magnitude of the magnetic fields there is up to $90 \mathrm{nT}$ (Fig. 7). When we compare the total field maps (Fig. 7) to the aeromagnetic map (Fig. 1), we see very good agreement not only in the location of crustal anomalies but also in the magnitude of the corresponding magnetic field.

\subsection{Alternative methods and results for the Austrian region}

We have further calculated the crustal field in the considered region (Austria) following three additional distinct methods:

a) Calculation of "model bias" for the whole period of study (2000-2012), i.e., the average of the residuals of the global model for the Austrian region at $3 \mathrm{~km}$ altitude;

b) Subtracting the core field predicted by a given global model from the measurements at given places and times;

c) Using directly the lithosphere field coefficients (from $L_{c}$ to $L_{\max }$ ) of global models.
These methods and the respective results are described in the following sections.

\subsubsection{Using the "model bias"}

For each observatory/repeat station, the average value of the residuals time series for the whole period (20012012) is calculated. These averages are subtracted from the respective residuals series, thus recovering the nonmodeled external field for the observatory/repeat station (Duka et al., 2016). The values of such external field are almost the same (a few nanoteslas) for the whole region due to the limited geographical extension. This can be shown for all models, but we present here the result for the POMME-9 model (Fig. 8). The external field is almost the same in all the sites of the Austrian repeat station network.

The averaged values of the "model bias" series recovered for every site of the Austrian network should represent the nonmodeled internal field, almost entirely of crustal origin (Duka et al., 2016). Therefore, we have plotted the respective maps for the POMME-9 model (Fig. 9). It can be seen that the anomalies initially identified in the eastern and central part of Austria are somehow recovered by the bias method, although not as satisfactorily as the residuals method discussed in Section 4.2. The method applied to the other models gives results of similar quality (maps are not shown here). 


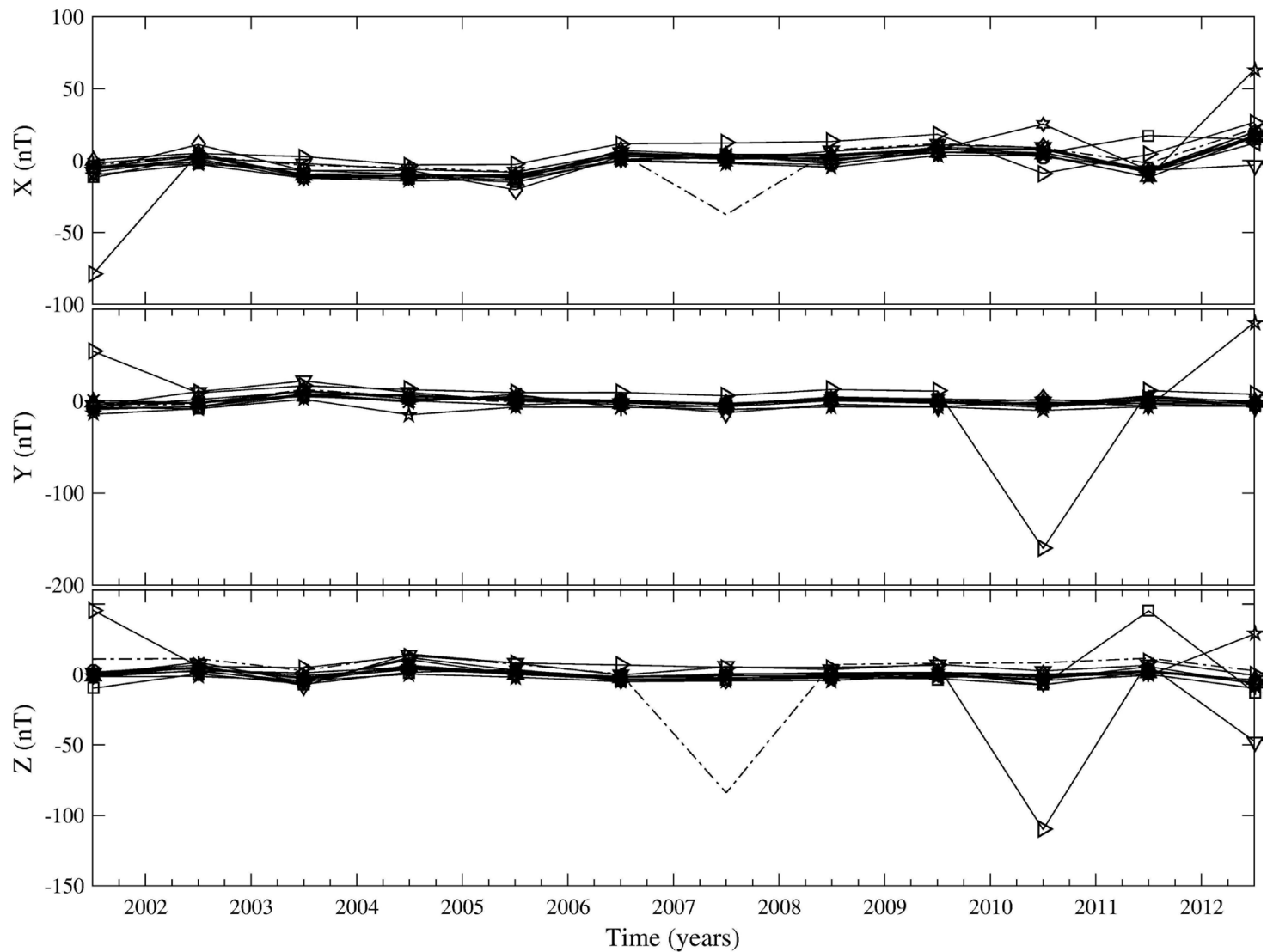

Figure 8: Reduced (by subtracting the average) residuals of geomagnetic field values measured at 13 points of the Austrian Repeat Station Network and the respective values predicted by the POMME-9 model (maximum degree 133) for the time period 2000-2012, The thicker curve represents the Kobenzl (WIK) Observatory.

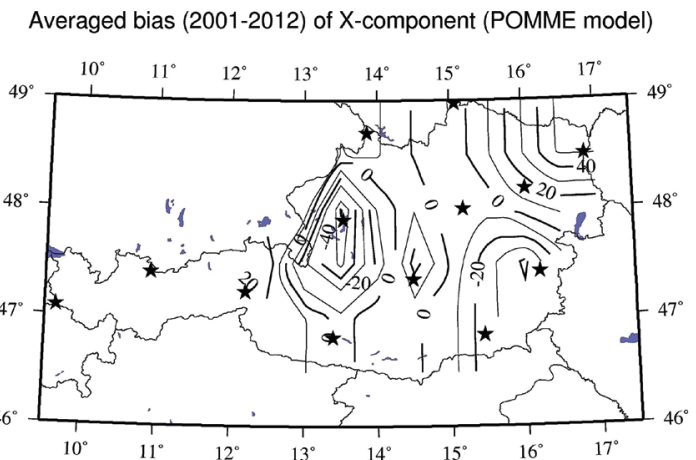

Averaged bias (2001-2012) of Z-component (POMME model)

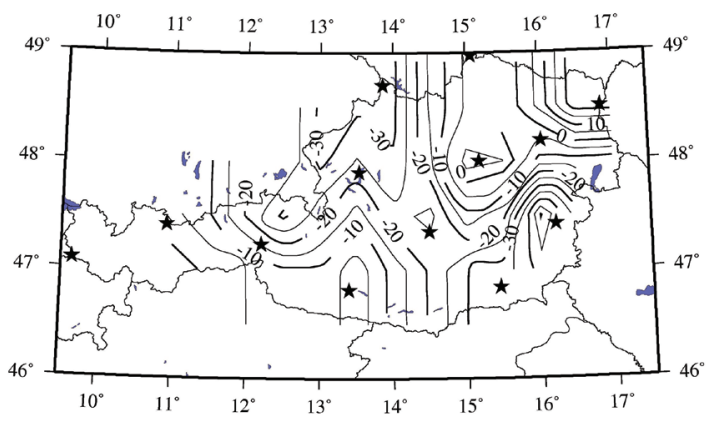

Averaged bias (2001-2012) of Y-component (POMME model)

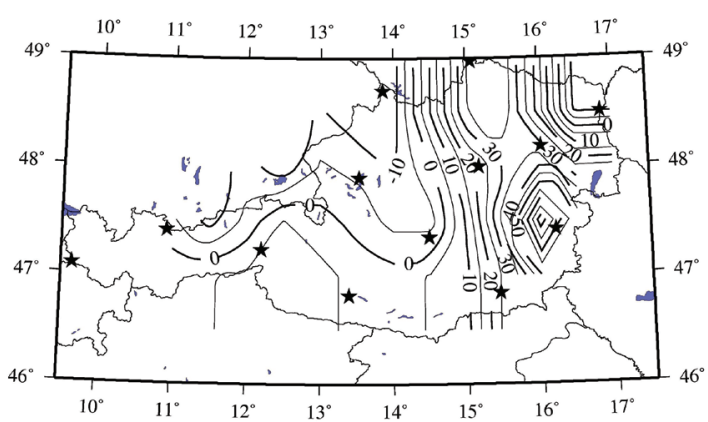

Averaged bias (2001-2012) of total field (POMME model)

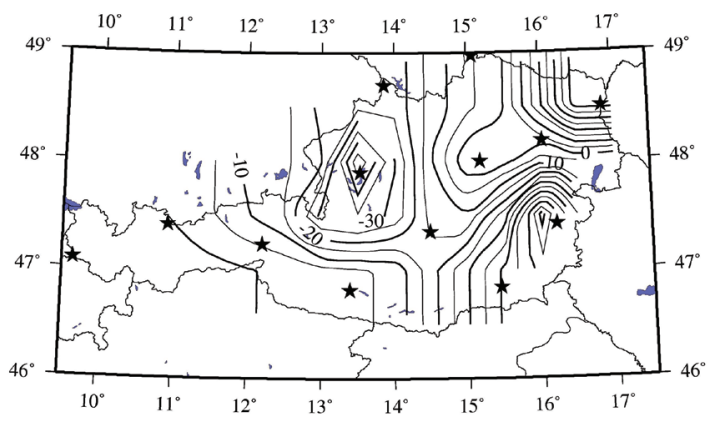

Figure 9: Maps of the averaged bias for the POMME-9 model calculated for the whole period 2001-2012. Data are in nanoteslas (nT). Observatories and repeat stations are marked with black stars. 


\subsubsection{Subtracting the core field}

The subtraction of the values of the geomagnetic core field predicted by a global model (up to $L_{c}$ ) for a given place from the values of the observed geomagnetic field at the same place represent in principle the crustal signal. Recall that the values given for each observatory/repeat station are annual means. Hence, the external field signal is effectively removed. Consequently, the residuals obtained should represent the static crustal field.

The crustal field is calculated as the differences between the observed field and core field of two models (EMM2015 and POMME-9) for epoch 2010 at the 13 points of Austrian Repeat Station Network. Maps of residuals for the $X$ and $Y$ components are shown in Fig. 10, while Fig. 11 displays the corresponding maps for $Z$ and the total field $F$. It is obvious that the maps of the crustal field are almost the same for both models. This means that the field recovered by this method has a static nature.

\subsubsection{Crustal field obtained directly from global models}

Each global model gives the crustal field separately. For the POMME-9 model, the crustal field is calculated by the spherical harmonics from degree 15 to degree 133 at regular $0.25^{\circ} \times 0.25^{\circ}$ grids covering Austria at the $3 \mathrm{~km}$ altitude (Fig. 12). The crustal field for the model EMM2015 is calculated by the spherical harmonics from degree 16 to degree 720 over the same grid at the same latitude (Fig. 13). The EMM2015 model seems to represent much better the geomagnetic field of crustal origin. However, there appear some anomalies that are not observed from the aeromagnetic data. This means that the results of the EMM2015 model should be considered with some caution.

\section{SV for the Austrian region}

We study the SV only for the Austrian territory. The SV for all the field components $(X, Y, Z$, and $F)$ is calculated as the difference between successive years of the time series (Wardinski and Holme, 2011)

$$
\left.\frac{d X}{d t}\right|_{T}=X(T)-X(T-1) .
$$

where $T$ is a given moment in time. The fact that the data of the repeat station network are reduced to the middle of a given year means that the external contributions of periods $<1$ year to the data are almost removed from the SV. In addition, in these differences represented in Eq. (6), the crustal contribution due to remanent magnetization is practically removed. Therefore, we can conclude that the series of SV represents the time changes of internal origin, mainly of core origin.

The calculation of the SV is problematic because the number of observatories/repeat station changes considerably during the period considered in this study. To cope with this problem, we have calculated the SV over the grid constructed for the ST model. The same grid was used for the global models as well. The EMM2015 model provides the SV directly, while for the normal (ST) field, the CHAOS-5, and the POMME-9 models, the SV is calculated following Eq. (6). For the global models, the field components and total field are initially calculated at the middle of each year. The SV is then calculated for
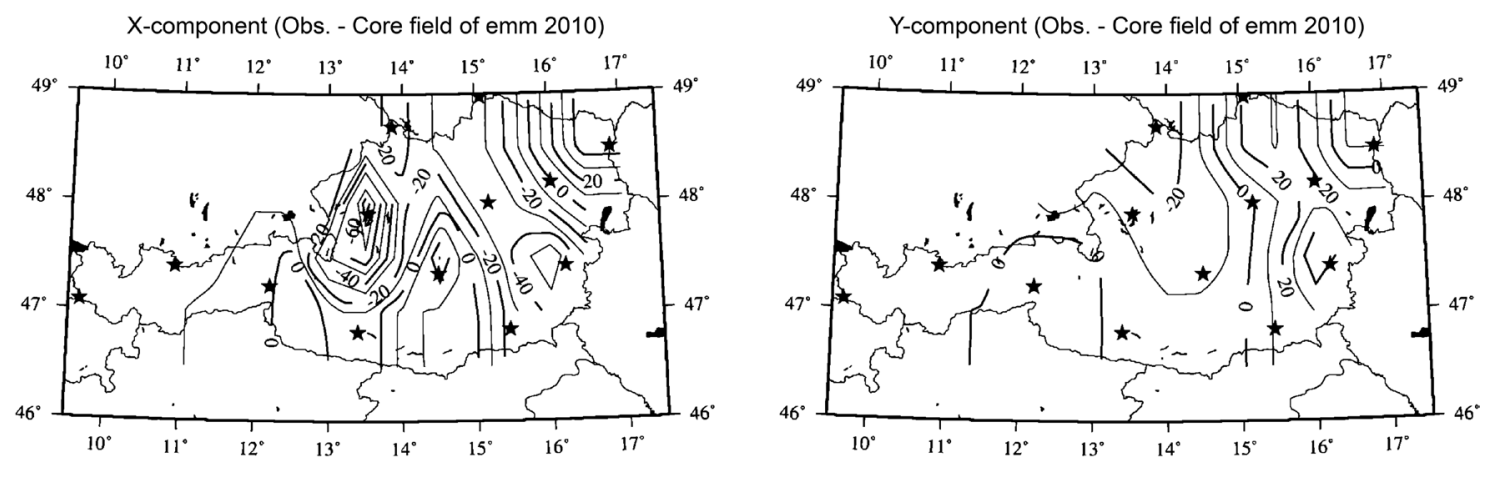

X-component (Obs. - Core field of Pomme 2010)
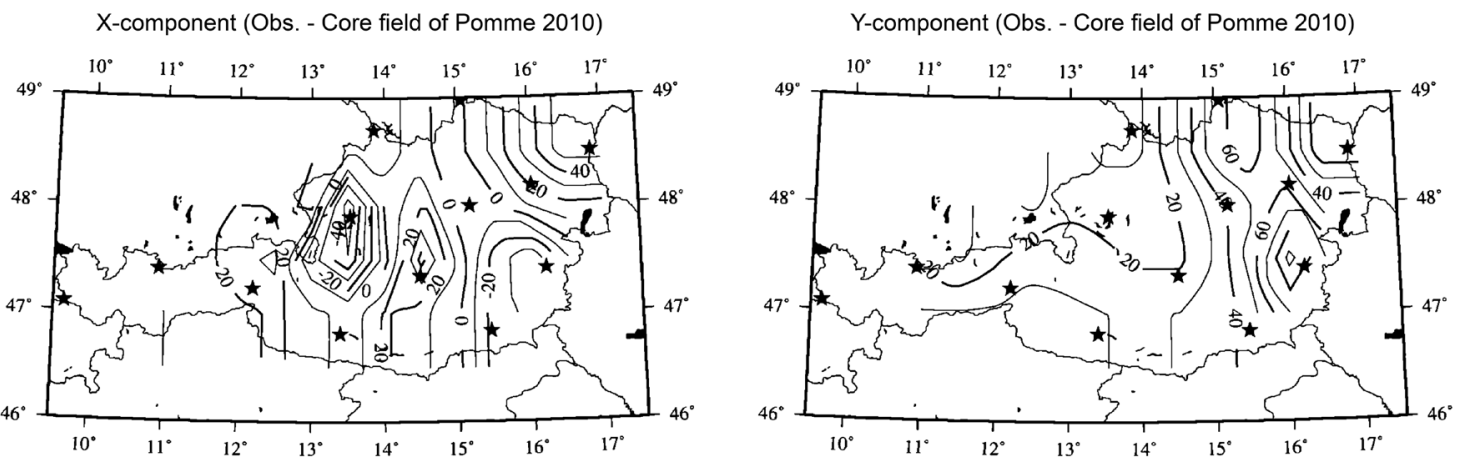

Figure 10: Plots of the $X$ and $Y$ components of the crustal field calculated as the differences between the observed field values and the respective values of core field given by the EMM2015 and POMME-9 models. The data are in nanoteslas (nT). Observatories and repeat stations are marked with black stars. 

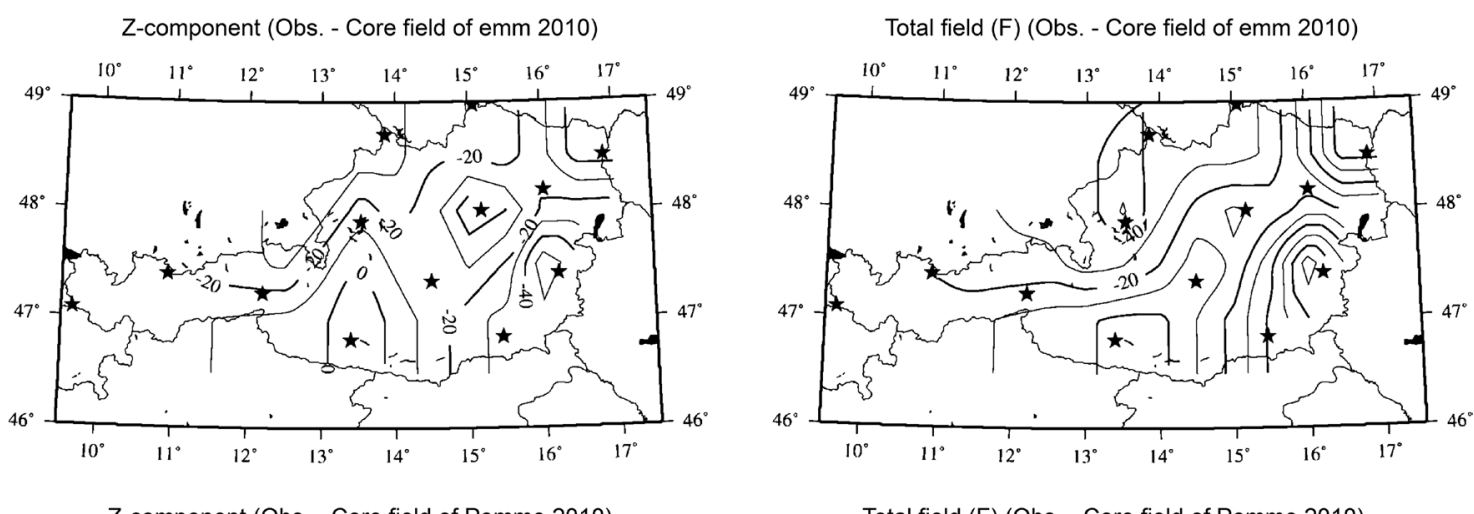

Z-component (Obs. - Core field of Pomme 2010)
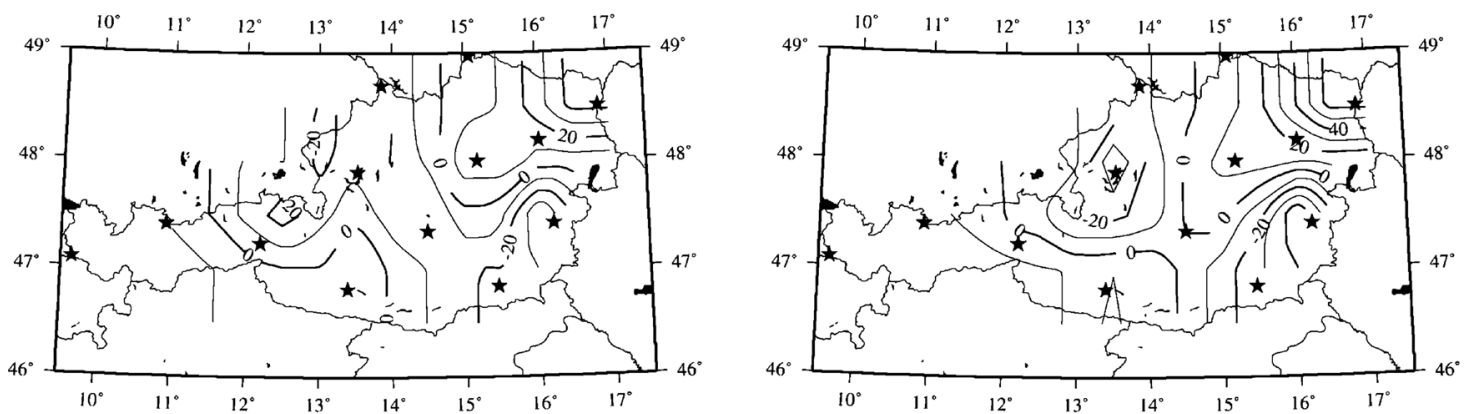

Figure 11: Plots of the $Z$ component of the crustal field and the total field $F$ calculated as the differences between the observed field values and the respective values of core field given by the EMM2015 and POMME-9 models. The data are in nanoteslas (nT). Observatories and repeat stations are marked with black stars.
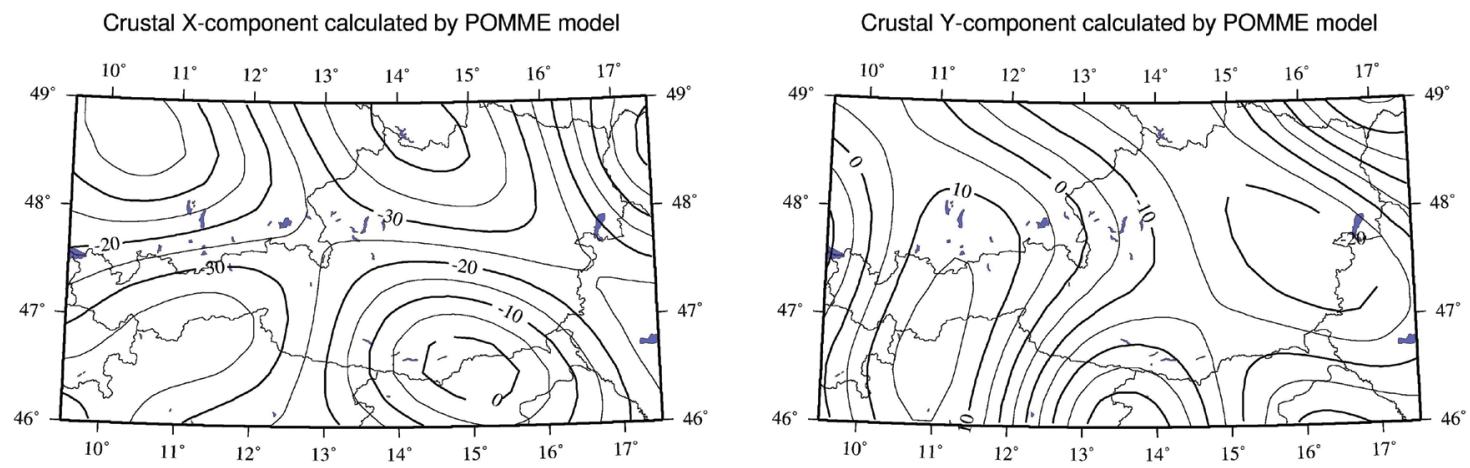

Crustal Z-component calculated by POMME model
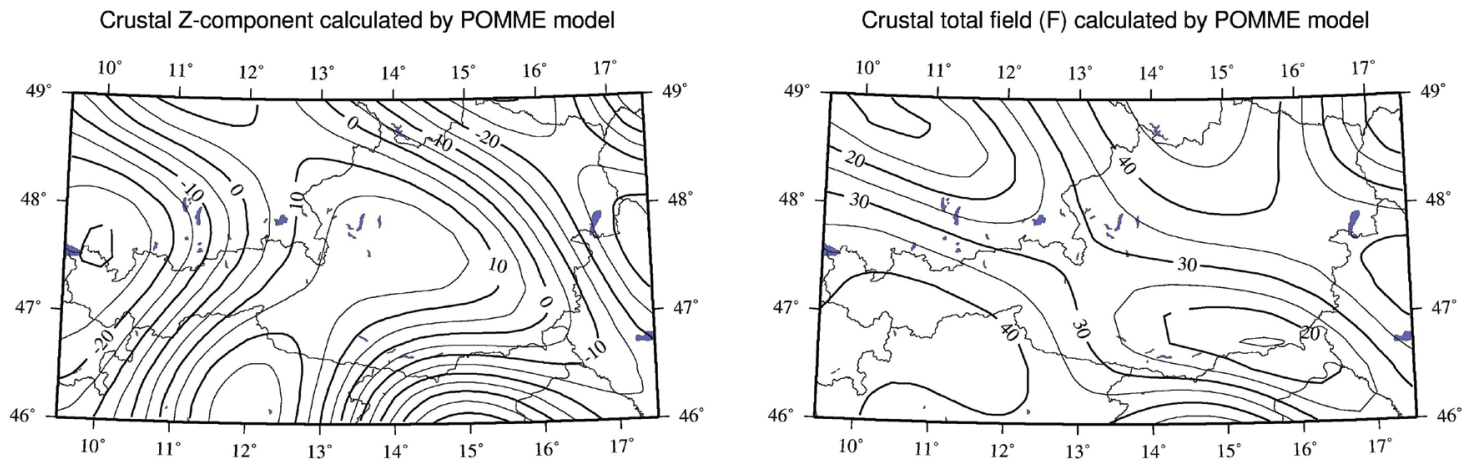

Figure 12: Plots of the crustal field calculated directly by the POMME-9 model (from $L_{c}=15$ to $L_{\max }=133$ ) at $3 \mathrm{~km}$ altitude. Data are in nanoteslas (nT).

each pair of years (2002 - 2001, 2003 - 2002, and so on) over the same grid of points for the three models. We present here the plots for the epoch 2006 (calculated as 2006 - 2005). We show here the plots for the ST and the POMME-9 models (Figs. 14 and 15).
The SVs of the geomagnetic field calculated by the regional or global models are very similar in magnitude over the whole territory of Austria (Figs. 14 and 15). Because of the presence of a limited number of points $(n=14)$ of the repeat station network, the maps present 

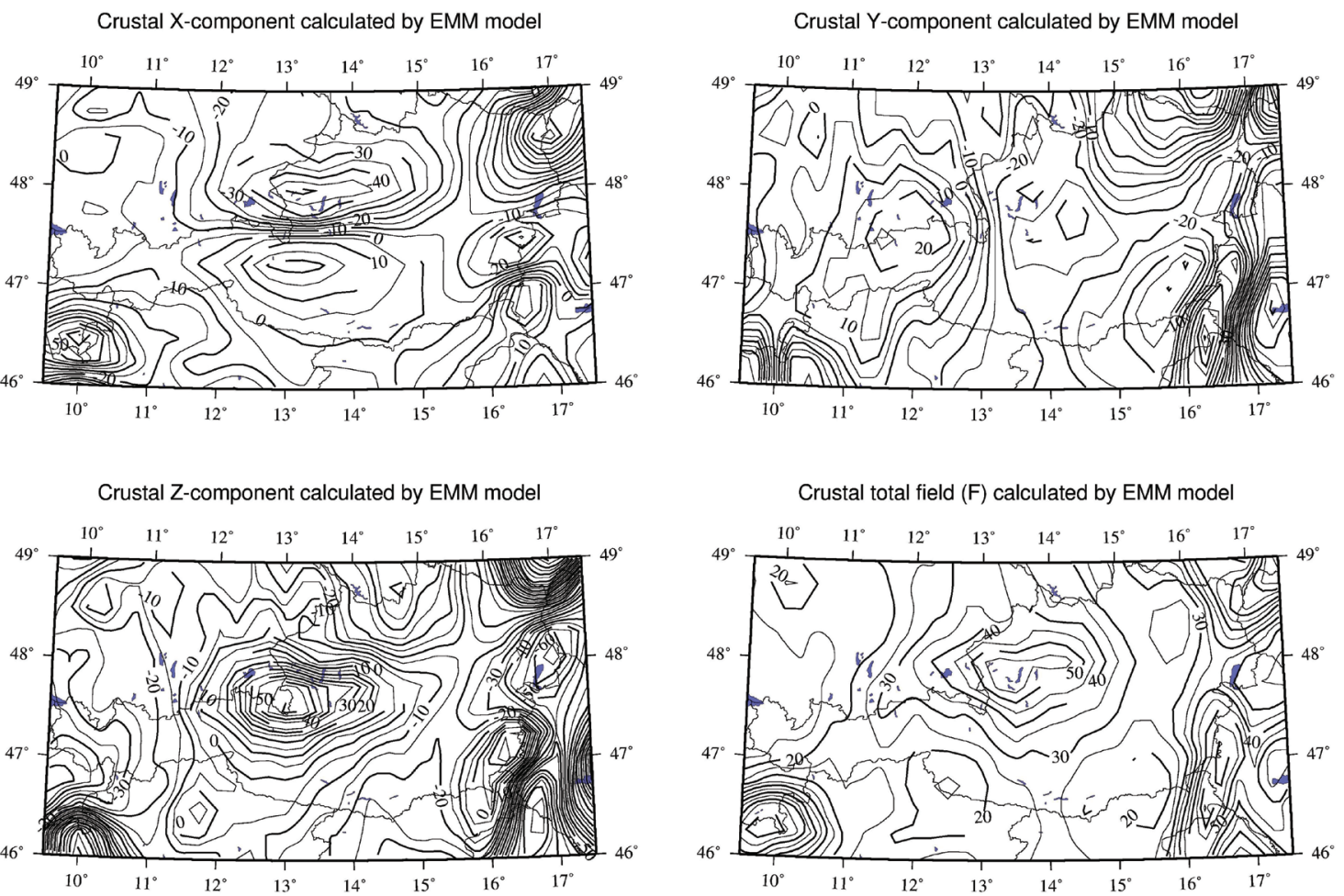

Figure 13: Plots of the crustal field calculated directly by the EMM2016 model (from $L_{c}=16$ to $L_{\max }=720$ ) at $3 \mathrm{~km}$ altitude. Data are in nanoteslas (nT).

SV of NORMAL Field 2006 (X-component)

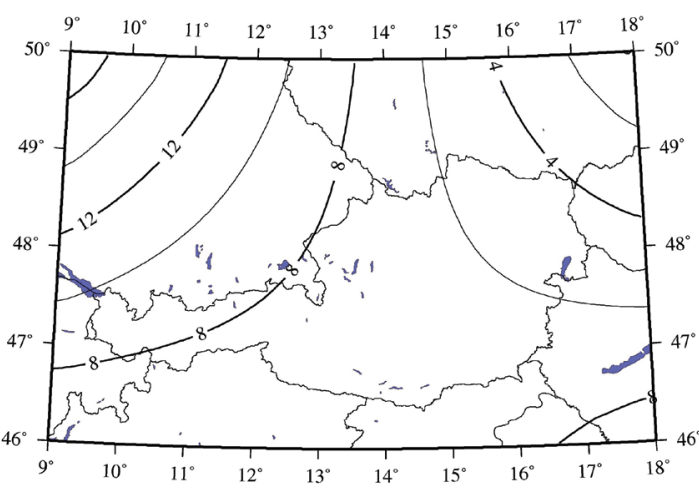

SV of NORMAL Field 2006 (Z-component)

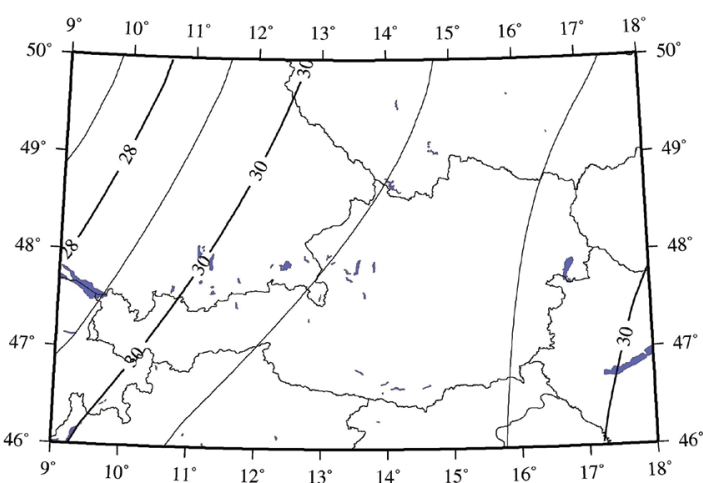

SV of NORMAL Field 2006 (Y-component)

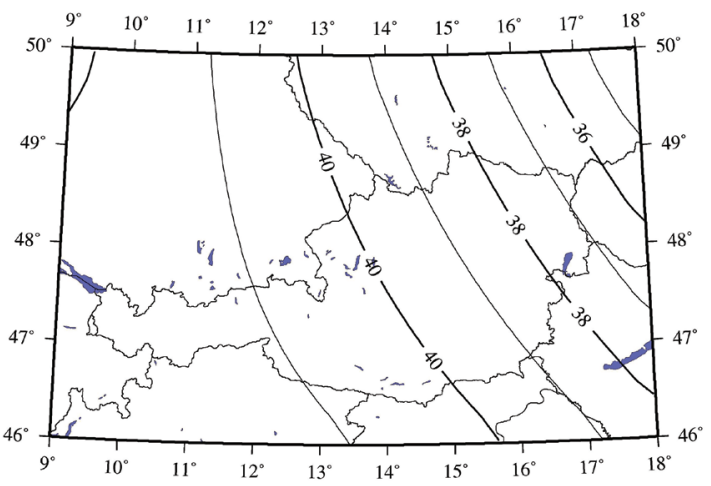

SV of NORMAL Field 2006 (total field F)

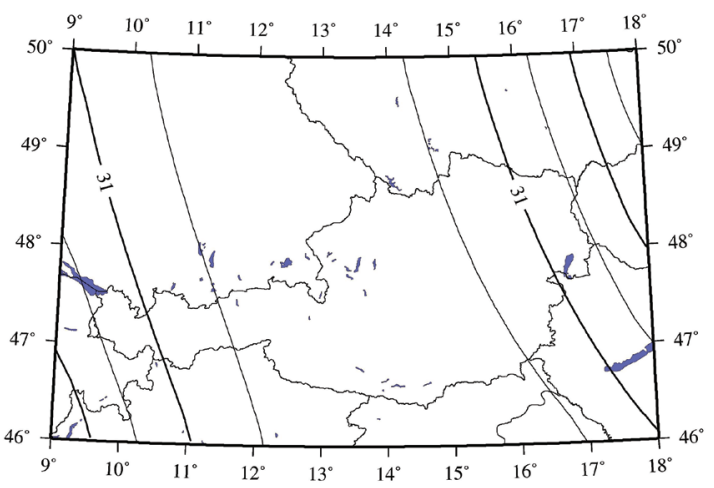

Figure 14: Maps of SV resulting from the normal field (ST) model (2006 - 2005). Data are in nanoteslas per year (nT/yr).

an uncomplicated picture of the SV. But the order of the values are almost the same even when the calculations are carried out for the $3 \mathrm{~km}$ altitude, as can be seen from Tables 2 and 3 . Figure 16 shows the curves for the real
SVs measured between consecutive repeat station campaigns. We noticed that there are very small changes in the SVs between different points of the repeat station network, apart from Station $4\left(48.494^{\circ} \mathrm{N} ; 16.857^{\circ} \mathrm{E}\right)$, 
SV of POMME Field 2006 (X-component)

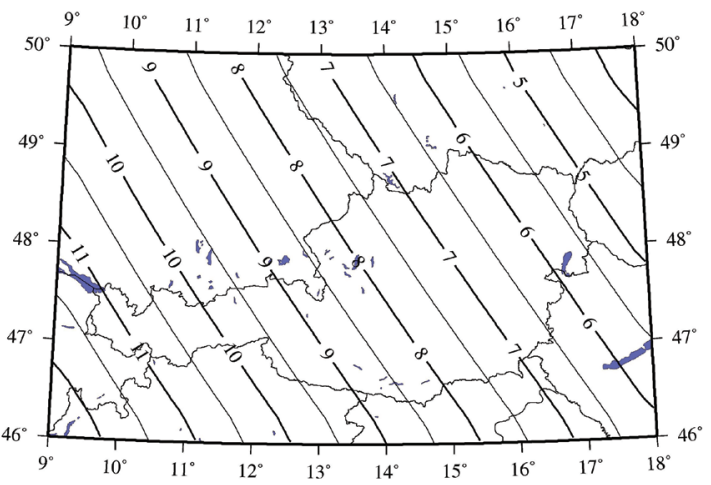

SV of POMME Field 2006 (Z-component)

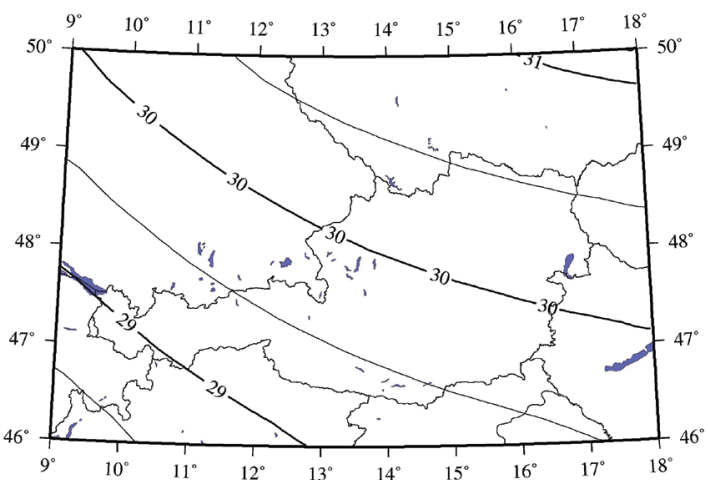

SV of POMME Field 2006 (Y-component)

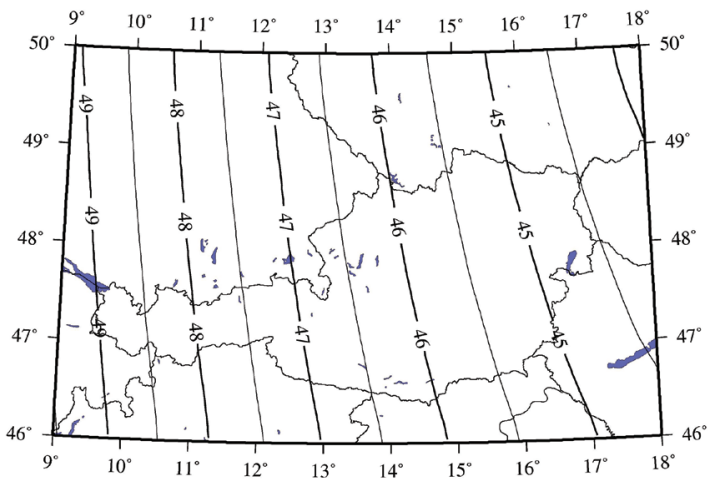

SV of POMME Field 2006 (total field F)

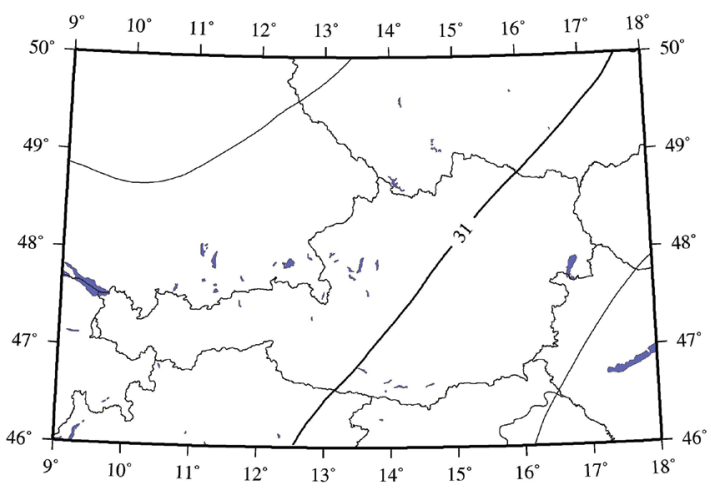

Figure 15: Maps of SV resulting from the POMME-9 model (2006 - 2005). Data are in nanoteslas per year (nT/yr).

\begin{tabular}{|c|c|c|c|c|c|c|c|c|c|c|c|c|c|c|c|c|c|c|c|c|c|c|c|}
\hline \multirow{2}{*}{\multicolumn{2}{|c|}{$\begin{array}{l}\text { Year } \\
\text { Model }\end{array}$}} & & \multicolumn{4}{|c|}{$2002-2001$} & \multicolumn{5}{|c|}{$2003-2002$} & \multicolumn{4}{|c|}{$2004-2003$} & \multicolumn{4}{|c|}{$2005-2004$} & \multicolumn{4}{|c|}{$2006-2005$} \\
\hline & & & $x$ & $Y$ & $\mathbf{Z}$ & $F$ & $\mathbf{x}$ & $\mathbf{Y}$ & $\mathbf{Z}$ & $\mathbf{F}$ & & $\mathrm{X}$ & $\mathbf{Y}$ & $\mathbf{Z}$ & $\mathbf{F}$ & $\mathbf{x}$ & $Y$ & $\mathbf{Z}$ & $F$ & $\mathbf{x}$ & $\mathbf{Y}$ & $\mathbf{Z}$ & $F$ \\
\hline \multicolumn{3}{|c|}{ Normal (ST) } & 11.4 & 29.9 & 30 & 32.4 & 10.8 & $\begin{array}{ll}8 & 31.9\end{array}$ & 929. & $\begin{array}{ll}.9 & 32 \\
\end{array}$ & & 10.43 & 34.12 & 29.9 & 31.9 & 9.8 & 30 & 29.7 & 31.5 & 9.3 & 38.2 & 29.6 & 31.3 \\
\hline \multicolumn{3}{|c|}{ CHAOS-5 } & 1.3 & 40.4 & 35.4 & 33 & 6.4 & 30.7 & 749. & 9.948 & & 17.13 & $36.4 \quad 1$ & 19.5 & 25.5 & 6.6 & 36.9 & 37.2 & 36.9 & 3.6 & 35.5 & 24.2 & 23.9 \\
\hline \multicolumn{3}{|c|}{ POMME-9 } & -0.3 & 40.5 & 33.3 & 30.4 & 8.2 & 28.8 & 38 & 38 & & 9.5 & $\begin{array}{ll}36.6 \quad 3 \\
-1\end{array}$ & 36.5 & 37.5 & 5.1 & 45.4 & 32.2 & 31.9 & 3.7 & 30.1 & 27.5 & 26.9 \\
\hline \multicolumn{3}{|c|}{ EMM2015 } & 6.4 & 35.2 & 37.2 & 39.2 & 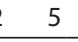 & 34.6 & 636. & $5.8 \quad 36$ & & 4.5 & $33.7 \quad 3$ & 33.2 & 32.4 & 7.1 & 33.8 & 31.1 & 31.7 & 9.9 & 36.1 & 28.8 & 31 \\
\hline \multicolumn{4}{|c|}{$2007-2006$} & \multicolumn{4}{|c|}{$2008-2007$} & \multicolumn{4}{|c|}{$2009-2008$} & \multicolumn{4}{|c|}{$2010-2009$} & \multicolumn{4}{|c|}{$2011-2010$} & \multicolumn{4}{|c|}{$2012-2011$} \\
\hline $\mathbf{X}$ & $\mathbf{Y}$ & $\mathbf{Z}$ & $F$ & $\mathrm{X}$ & $Y$ & $\mathbf{Z}$ & $\mathbf{F}$ & $\bar{x}$ & $\mathbf{Y}$ & $\mathbf{Z}$ & $\mathbf{F}$ & $\mathbf{X}$ & $\mathbf{Y}$ & $\mathbf{Z}$ & $\mathbf{F}$ & $\mathbf{X}$ & $\mathbf{Y}$ & $\mathbf{Z}$ & $F$ & $\mathbf{X}$ & $\mathbf{Y}$ & $\mathbf{Z}$ & $\mathbf{F}$ \\
\hline 8.7 & 40.2 & 29.5 & 31 & 8.2 & 42.4 & 29.5 & 30.9 & 7.7 & 44.3 & 29.4 & 30.5 & $\begin{array}{ll}5 & 7.1\end{array}$ & 40.4 & 29.3 & 30.3 & 6.6 & 48.5 & 29.2 & 30 & 6.1 & 50.6 & 29.2 & 29.9 \\
\hline 15.1 & 38.5 & 24.6 & 29.4 & 11 & 38 & 26.6 & 29.4 & 4.8 & 46.1 & 24.9 & 25.3 & $\begin{array}{ll}3 & -1.2\end{array}$ & 50.7 & 34.8 & 31.9 & 11.2 & 44.5 & 28.3 & 31.3 & -17.6 & 54.9 & 40.9 & 30.5 \\
\hline 10.8 & 37.9 & 28.1 & 30.7 & 12.4 & 34.2 & 27.5 & 30.8 & 11.3 & 47.9 & 29.9 & 32.7 & 4.1 & 59.5 & 30 & 29.9 & 0.2 & 42.4 & 26.2 & 25 & 8.6 & 40.7 & 30.5 & 32.1 \\
\hline 11.4 & 39.4 & 27.4 & 30.3 & 8.4 & 43.4 & 26.7 & 28.6 & 4.8 & 47.4 & 29.1 & 29.3 & 3.6 & 48.1 & 29.7 & 29.3 & 4.5 & 49.3 & 32.8 & 32.6 & 5.4 & 49.1 & 31.7 & 32.1 \\
\hline
\end{tabular}

Table 2: Average values (in nanoteslas per year [nT/yr]) of SV for the Austrian region ( $0 \mathrm{~km}$ altitude) according to different models.

\begin{tabular}{|c|c|c|c|c|c|c|c|c|c|c|c|c|c|c|c|c|c|c|c|c|c|c|c|}
\hline \multirow{2}{*}{\multicolumn{2}{|c|}{$\begin{array}{l}\text { Year } \\
\text { Model }\end{array}$}} & \multicolumn{5}{|c|}{$2002-2001$} & \multicolumn{4}{|c|}{$2003-2002$} & \multicolumn{4}{|c|}{$2004-2003$} & \multicolumn{5}{|c|}{$2005-2004$} & \multicolumn{4}{|c|}{$2006-2005$} \\
\hline & & $\bar{x}$ & $Y$ & $Y$ & $\mathbf{Z}$ & $\mathbf{F}$ & $x$ & $\mathbf{Y}$ & $\mathbf{Z}$ & $\mathbf{F}$ & $\mathbf{X}$ & $\bar{Y}$ & $\mathbf{Z}$ & $\mathbf{F}$ & $\mathbf{x}$ & $\bar{Y}$ & & $\mathbf{Z}$ & $\mathbf{F}$ & X & $\mathbf{Y}$ & $\mathbf{Z}$ & $\mathbf{F}$ \\
\hline \multicolumn{2}{|c|}{ lormal (ST) } & 1. & 29 & 9.8 & 5 & 32.3 & 10.8 & & & & 10 & 34 & & & 9 & 3 & & 29.7 & 31.5 & 9.3 & 38 & 9.6 & 31.2 \\
\hline \multicolumn{2}{|c|}{ AOS-5 } & & 10 & 0.4 & 55. & 33 & & & & & & & & & & & & & & & & & \\
\hline \multicolumn{2}{|c|}{ MME-9 } & & 46 & 6.3 & 5. & 34 & 0. & & & & & & & & 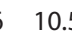 & & & & & & & & \\
\hline & 015 & & & & 7.6 & 37. & & & & & & & & & & 3 & & & 31 & & & & \\
\hline \multicolumn{4}{|c|}{$2007-2006$} & \multicolumn{4}{|c|}{$2008-2007$} & \multicolumn{4}{|c|}{$2009-2008$} & \multicolumn{4}{|c|}{$2010-2009$} & \multicolumn{4}{|c|}{$2011-2010$} & \multicolumn{4}{|c|}{$2012-2011$} \\
\hline $\mathrm{X}$ & $\mathbf{Y}$ & - & $\mathbf{F}$ & $\mathrm{X}$ & $\mathbf{Y}$ & $\mathbf{Z}$ & $\mathbf{F}$ & $\boldsymbol{\lambda}$ & 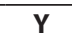 & $\mathbf{Z}$ & $\mathbf{F}$ & $\mathbf{X}$ & $\bar{Y}$ & - & $\mathbf{F}$ & $\boldsymbol{X}$ & $\mathbf{Y}$ & $\mathbf{Z}$ & 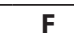 & $x$ & - & Z & $\mathbf{F}$ \\
\hline 8.7 & 0.1 & & & 8.2 & 42. & & & & & & & 7.1 & & & & & & & & & & & 9.9 \\
\hline r & 8.5 & .6 & 9.4 & 11 & 37. & 20 & & & & & & -1. & & & & 11 & & 5 & 31 & -17.6 & & & \\
\hline 5 & & & & 12.4 & & 27 & & & & & & 4. & & & & & & $t$ & & & & & 27 \\
\hline 1.4 & 39.3 & . 3 & .2 & 5.3 & 43.3 & 26.8 & 28.5 & 4.8 & 47.4 & 29.1 & 29.2 & 3.6 & 48.1 & 29.7 & 29.3 & 4.5 & 49.3 & 32.7 & 32 & 1.9 & 49.1 & 31.6 & 32 \\
\hline
\end{tabular}

Table 3: Average values (in nanoteslas per year [nT/yr]) of SV for the Austrian region (3 km altitude) according to different models. 

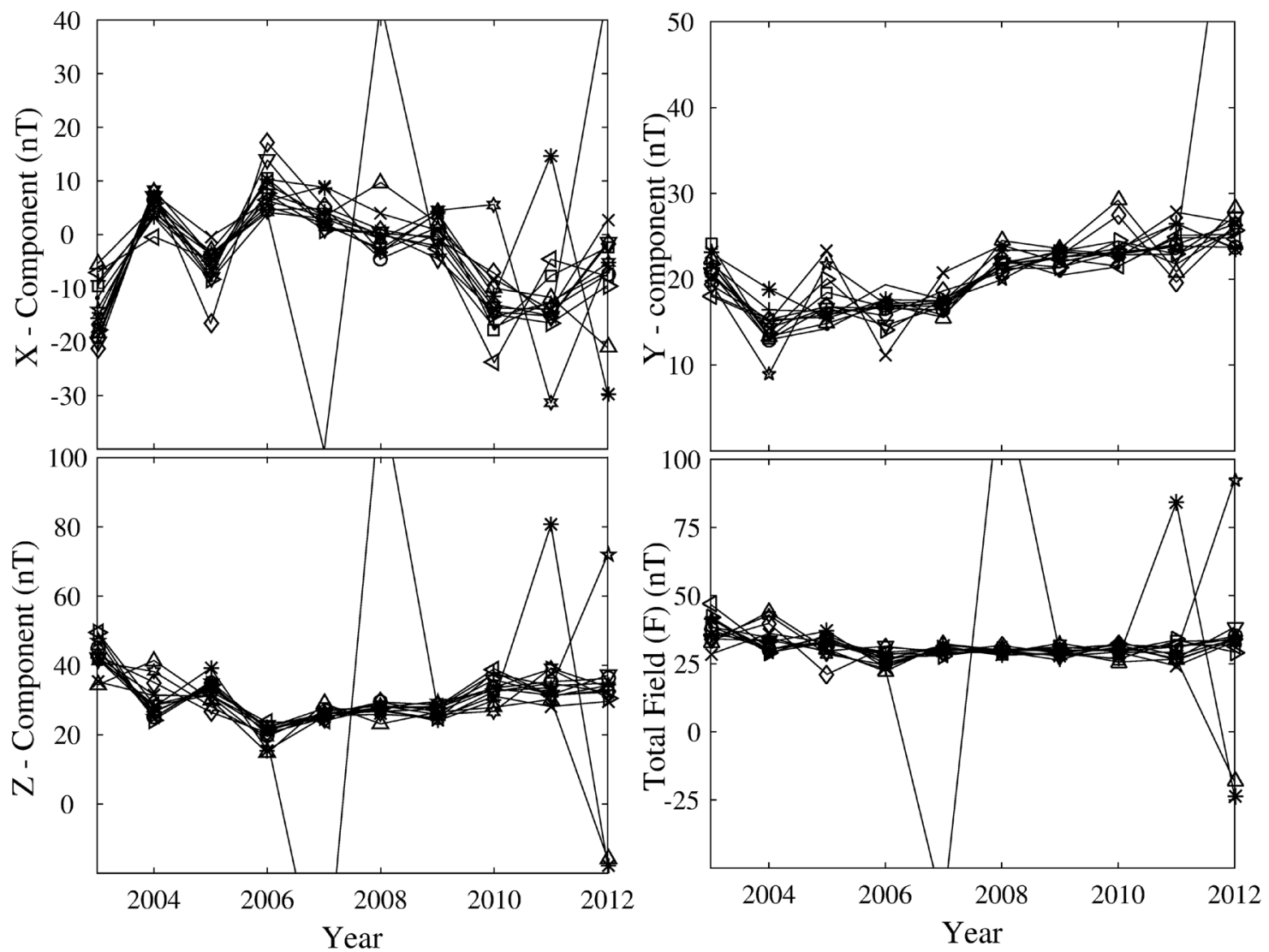

Figure 16: SV of the components of the geomagnetic field for the whole period (2003-2012) at 14 points of the Austrian Repeat Station Network.

where abnormal values belong to the year 2007 for $X$ and $Z$ components (Fig. 16).

The SV defined by the Austrian repeat stations seems to be better reproduced by the ST model rather than the global models. This example shows that the global models do not capture the SV of very small portions of the surface of the Earth. We support this claim by the results shown in Fig. 16. It can be seen that the SV values are very similar for the majority of the repeat stations but, time to time, there are significant differences. The ST model captures this changes; however, we cannot conclude on the smoothness of the SV isolines for the maps produced by each of the models. Note that although the outliers may be due to measurement errors or local disturbances, they are eliminated from the data set and do not interfere with our analysis.

\section{Discussions and conclusions}

A comparison between different approaches for calculating the crustal field over the Austrian region shows that including data from neighborhood countries is of crucial importance to obtain results that are more reliable. The accuracy of the geomagnetic measurements is highly improved, and measurement errors have negligible effects (Lowrie, 2007). The local crustal anomalies estimated by the residual of the regional (ST) model, i.e., by the differences between the observed values and the respective values predicted by the model, show different patterns for different years and different geomagnetic field components. We think that this problem is related to differences in data availability over time. However, the more pronounced anomalies in the central and eastern parts of the Austrian region represent a common feature for all years and components. This anomaly is more evident for the $Y$ and $Z$ components. The normal field pattern (dotted lines, Figs. 4-7) of the total field $(F)$ is more or less similar to that of the $Z$ component.

Similar features are visible in the crustal anomaly maps estimated by the residuals of the global models (CHAOS-5, EMM2015, and POMME-9) for $0 \mathrm{~km}$ altitude or $3 \mathrm{~km}$ altitude. Little variations are observed over time. All maps have common features: two pronounced anomalies in the eastern part of Austria, and one in the central region of Austria. These features are evident for CHAOS-5 and POMME-9 models, especially for the $Y$ and $Z$ components, as well as the total field. Comparing the results from the different models, we can conclude that they are more or less similar to those of the regional ST model.

Regarding the crustal field anomalies estimated by the "averaged biases" of different global models at the 13 points of the Austrian repeat station network (time period 2001-2012), there are common patterns similar to those of residuals maps. The eastern anomaly appears to be the superposition of two smaller anomalies, and this is more visible for the POMME-9 model. Furthermore, crustal anomalies are more visible for the $X$ and $Y$ components (followed by the total field F). 
Crustal field anomalies estimated by the differences of the observed values at the 13 points of the Austrian repeat station network and the respective values predicted by the global models (CHAOS-5, EMM2015, and POMME-9) of the core field show small discrepancies relative to each other, indicating that these residuals have static crustal nature.

Crustal fields calculated directly by the global models (spherical harmonic from $L_{c}+1$ to $L_{\text {max }}$ ) are quite homogeneous in the case of the POMME-9 model. The EMM2015 model, which goes up to $L_{\text {max }}=720$, provides a higher resolution in wavelength $(56 \mathrm{~km})$ with respect to the other models. This model gives additional anomalies in the central part of Austria.

The SV for the Austrian region is calculated in the grid described in the respective section by regional (ST) and global (CHAOS-5, EMM2015, and POMME-9) models. The SV patterns of the ST model show moderate variation over the whole region, but unfortunately, we cannot compare these with aeromagnetic data. Global models yield smoother SV patterns. The ST model seems to describe the SV on a regional scale better than the global model. Despite the limited geographical extension of the Austrian region, it is large enough to assume more complicated SV patterns than those produced by the global models and is possibly similar to those obtained by the ST model. However, this conclusion remains speculative.

\section{Acknowledgment}

This study was supported by the bilateral project "Using ground and satellite data to study the variations of the geomagnetic field over Austria and Albania", included in the Annex 1 (AL 04/2015-16) of the Protocol of the 2nd Meeting of the Austrian-Albanian Joint Commission for Scientific and Technological Co-operation.

\section{References}

Achache, J., Abtout, A., and Le Mouël, J.-L., 1987. The downward continuation of Magsat Crustal Anomaly Field over Southeast Asia. Journal of Geophysical Research, 92, 11584-11596.

Alldredge, L.R., 1981. Rectangular Harmonic Analysis applied to the geomagnetic field. Journal of Geophysical Research, 86, 3021-3026.

Asgharzadeh, M.F., von Frese, R.R.B., and Kim, H.R., 2008. Spherical prism magnetic effects by GaussLegendre quadrature integration. Geophysical Journal International, 173, 315-333. https://doi. org/10.1111/j.1365-246X.2007.03692.x

Backus, G., Constable, C. and Parker, R., 1996. Foundations of Geomagnetism. New York, NY: Cambridge University Press.

Bloxham, J., and Gubbins, D., 1986. Geomagnetic fiels analysis IV - Testing the frozen flux hypothesis. Geophysical Journal of the Royal Astronomical Society, 84, 139-152.
Chiappini, M., Meloni, A., Boschi, E., Faggioni, O., Beverini, N., Carmisciano, C., Marson, I., 2000. Shaded relief magnetic anomaly map of Italy and surrounding marine areas. Annals of Geophysics, 43/5, 983-989. https://doi. org//10.4401/ag-3676

De Santis, A., Battelli, O., and Kerridge, D.J., 1990. Spherical cap harmonic analysis applied to regional field for Italy. Journal of geomagnetism and geoelectricity, 42, 1019-1036.

Duka, B., Duka, E. and Peqini, K., 2016. Recovering external contribution from the monthly mean series of a given geomagnetic observatory. Annals of Geophysics, 59/3, G0321. https://doi.org/10.4401/ag-6971

Duka, B., Gaya-Piqué, L. R., De Santis, A., Bushati, S., Chiappini, M. and Dominici, G., 2004. A geomagnetic reference model for Albania, Southern Italy and the Ionian Sea from 1990 to 2005. Annals of Geophysics, 47/5, 1609-1615.

Düzgit, Z. and Malin, S.R.C., 2000. Assessment of regional geomagnetic field modeling methods using a standard data set: spherical cap harmonic analysis. Geophysical Journal International, 141, 829-831.

Finlay, C.C., Olsen, N. and Tøffner - Clausen, L., 2015. DTU candidate field models for IGRF-12 and the CHAOS-5 geomagnetic field model. Earth, Planets and Space 67, 114. https://doi.org/10.1186/ s40623-015-0274-3

Haines. G.V., 1985. Spherical cap harmonic analysis. Journal of Geophysical Research, 90, B3, 2583-2591.

Jacobs, J. A. (ed.), 1991. Geomagnetism 4. Academic Press, London, $481 \mathrm{pp}$.

Khesin, B.E., Alexeyev, V.V. and Eppelbaum, L.V., 1996. Interpretation of Geophysical Fields in Complicated Environments. Kluwer Academic Publishers, Series: Modern Approaches in Geophysics, Boston - Dordrecht London, 368 pp.

Lowrie, W., 2007. Fundamentals of Geophysics, 2nd edition, Cambridge University Press, Cambridge, UK, 381 pp.

Macmillan, S., and Thomson, A., 2003. An examination of observatory biases during the Magsat and Ørsted missions. Physics of the Earth and Planetary Interiors, 135, 97-105. https://doi.org/10.1016/ S0031-9201(02)00209-1

Mandea, M. and Langlais, B., 2002. Observatory Crustal Magnetic Biases during MAGSAT and Oersted Satellite Missions. Geophysical Research Letters, 29/15, 8003. https://doi.org/10.1029/2001GL013693

Maus, S., 2010. An ellipsoidal harmonic representation of Earth's lithospheric magnetic field to degree and order 720. Geochemistry, Geophysics, Geosystems, 11, Q06015. https://doi.org/10.1029/2010GC003026.

Maus, S. and Haak, V., 2002. Is the Long Wavelength Crustal Magnetic Field Dominated by Induced or by Remanent Magnetization? Journal of Indian Geophysical Union, 6/1, 1-5.

Mayhew, M.A., 1979. Inversion of satellite magnetic anomaly data. Journal of Geophysics, 45, 119-128. 
Merrill, R. and Mcfadden, P.H., 1999. Geomagnetic polarity transitions. Reviews of Geophysics, 37, 201-226. https://doi.org/10.1029/1998RG900004

Nakagawa, I. and Yukutake, T., 1985. Rectangular harmonic analysis of geomagnetic anomalies derived from Magsat data over the area of the Japanese islands. Journal of Geomagnetism and Geoelectricity, 37, 957-77.

Nolte, H. J. and Hahn, A., 1992. A model of the distribution of crustal magnetization in central Europe compatible with the field of magnetic anomalies deduced from Magsat results. Geophysical Journal International, 111, 483-496.

O'Brien, M.S. and Parker, R.L., 1994. Regularized field modeling using monopoles. Geophysical Journal International, 118, 566-578.

Olsen, N. and Stolle, C., 2016. Magnetic Signatures of Ionospheric and Magnetospheric Current Systems During Geomagnetic Quiet Conditions - An Overview. Space
Science Reviews, 206, 5-25. https://doi.org/10.1007/ s11214-016-0279-7

Purucker, M.E., 1990. The computation of vector magnetic anomalies: a comparison of techniques and errors. Physics of the Earth and Planetary Interiors, 62, 231-245. Richter, P.H., 1995. Estimating Errors in Least - Squares Fitting. TDA Progress Report, 42-122.

Taylor, P.T. and Ravat, D., 1995. An interpretation of the Magsat anomalies of central Europe. Journal of Applied Geophysics, 34, 83-91. https://doi. org/10.1016/0926-9851(95)00015-1

Voigt, G-H., 1981. A mathematical Magnetospheric Field Model with independent physical parameters. Planetary and Space Science, 29, 1-20.

Wardinski, I. and Holme, R., 2011. Signal from noise in geomagnetic field modeling: de noising data for secular variation studies. Geophysical Journal International, 185, 653-662. https://doi. org/10.1111/j.1365-246X.2011.04988.x. 